\title{
ANALYTICAL SOLUTION OF MIXED BOUNDARY VALUE PROBLEMS USING THE DISPLACEMENT POTENTIAL APPROACH FOR THE CASE OF PLANE STRESS AND PLANE STRAIN CONDITIONS
}

\author{
S.K. DEB NATH \\ Institute for Materials Research, Tohoku University \\ JAPAN \\ E-mail: sankar20006@yahoo.com
}

\begin{abstract}
Two elastic plate problems made of duralumin are solved analytically using the displacement potential approach for the case of plane strain and plane stress conditions. Firstly, a one end fixed plate is considered in which the rest of the edges are stiffened and a uniform load is applied to the opposite end of the fixed end. Secondly, a plate is considered in which all of the edges are stiffened and a uniform tension is applied at its both ends. Solutions to both of the problems are presented for the case of plane stress and plane strain conditions. The effects of plane stress and plane strain conditions on the solutions are explained. In the case of stiffening of the edges of the plate, the shape of the plate does not change abruptly, which is clearly observed in both of the cases. For the plane strain condition, the plates become stiffer in the loading direction as compared to the plane stress condition. For the plane strain condition, there is a significant variation of the normal stress component, $\sigma_{z z}$ at different sections of the plate. The graphical results, clearly identify the critical regions of the plate for the case of the plane stress and plane strain condition.
\end{abstract}

Key words: analytical solution, displacement potential approach, plane stress and plane strain conditions, mixed boundary value problems.

\section{Introduction}

In the early nineteenth century, several theoretical approaches were developed to deal with the 2D isotropic material elements with simple boundary conditions [1-9]. The main limitations of these approaches are that they are not able to deal with the problems of isotropic materials with mixed boundary conditions. The reduction of the $2 \mathrm{D}$ elasticity problem under plane strain or plane stress conditions with the statement of the biharmonic problem is usually associated with the name of the Astronomer Royal George Biddel Airy [1]. In his paper [1] Airy considered a flexure of a finite rectangular beam as a 2D problem in the theory of elasticity. However, in [2] (section 144) Love expressed the displacement components corresponding to plane strain in terms of Airy's stress function [1]. Several methods have recently been developed to study the elasticity and thermoelasticity problems [10-19]. Vigak [10] proposed a method based on the integration of the differential equations of equilibrium of constructing exact solutions of two-dimensional problems of elasticity and thermoelasticity in the stresses for a rectangular region under prescribed forces on the boundary. The relaxation method developed by Southwell [19] for various problems of the theory of elasticity was also applied [20] to study the biharmonic problem for a rectangle. In the review article [21], Meleshko gave a historical overview of some topics related to the classical 2D biharmonic problem describing shortly 758 references because this problem arises in many physical studies concerning bending of clamped thin elastic isotropic plates, equilibrium of an elastic body under conditions of plane strain or plane stress. He elucidated some interesting points related to the history of the problem and gave an overview of some analytical approaches to its solution. Ogden and Isherwood [22] developed new formulations of the governing equations for finite plain-strain deformations of compressible isotropic elastic solids. Elasticity problems are usually formulated either in terms of deformation parameters or stress 
parameters. Among the existing mathematical models of plane boundary-value stress problems, the stress function approach [1] and the displacement formulation [23]. Recently, Patnaik [24] developed boundary conditions for strain formulations which is a single partial differential equation in terms of two displacement components. In [25], Patnaik et al. introduce the compatibility concepts in elasticity, finite element analysis, design optimization.

It is noted that the recent research and developments in using the displacement potential boundary approach [26-28] have generated renewed interest in the field of both analytical and numerical solutions of stress problems. In this paper, firstly, a one end fixed duralumin plate having stiffening at rest of other edges subjected to a uniform tension at its right lateral edge is solved analytically for the case of plane stress and plane strain conditions and the solutions for both of the cases are discussed in a comparative fashion. Secondly, a duralumin plate is considered; all of its edges are stiffened along the $x$-direction and uniform tension is applied at its both opposite ends; this problem is solved plane stress and plane strain conditions; the solutions of the problem are discussed for the case of plane stress and plane strain conditions.

\section{Numerical model of the problem}

Two problems are considered in the present research work. Firstly, a one end fixed plate made of duralumin is considered in which $\mathrm{AB}$ is rigidly fixed; $\mathrm{AD}$ and $\mathrm{BC}$ are stiffened along the $y$-direction; $\mathrm{CD}$ is stiffened along the $\mathrm{x}$ direction as shown in Fig.1. A uniform tension is applied at its right lateral edge, CD. Secondly, a stiffened plate EFGH made of duralumin is considered. Its two edges EH and FG are considered as stiffened along the $y$-direction; its other two edges EF and HG are also stiffened along the $x$ direction; a uniform tension is applied at its two opposite ends, EF and GH, respectively as shown in Fig.2. In the first and the second problem, the maximum intensity of the applied tensile stress at the tip of the plate is $50 \mathrm{MPa}$. The Young's' modulus and Poisson's ratio of duralumin (plate material) are taken as $69 \mathrm{GPa}$ and 0.335 , respectively, to solve the problems.

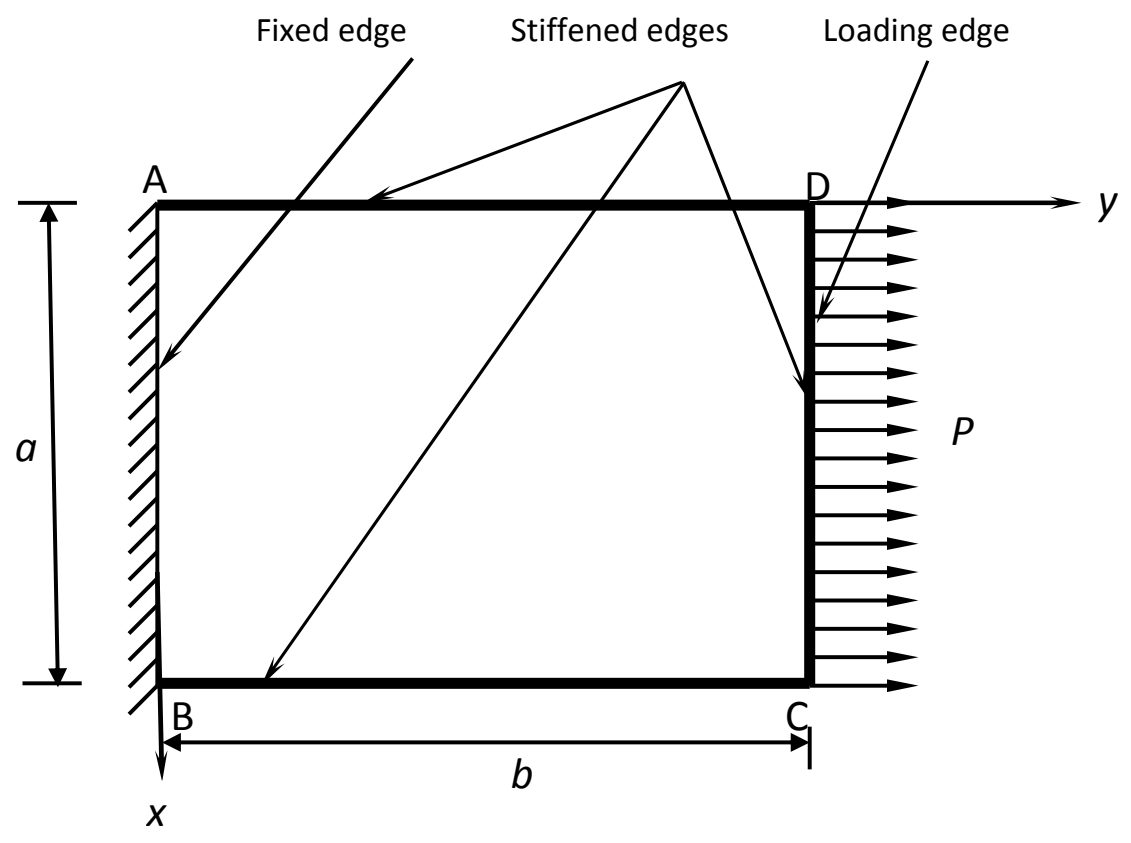

Fig.1. Geometry of a one end fixed stiffened duralumin plate subjected to uniform axial loading at its tip. 


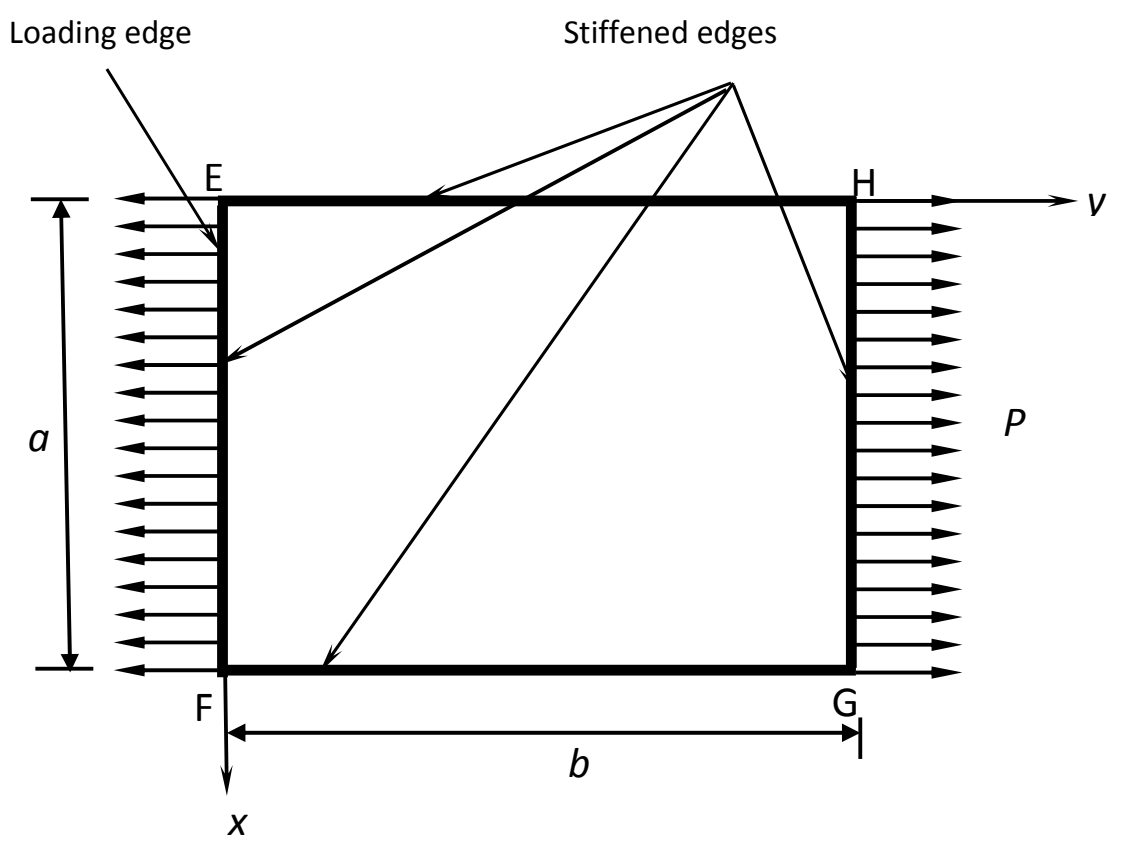

Fig.2. Geometry of a stiffened duralumin plate subjected to uniform axial loading at its two opposite ends.

\section{Displacement potential formulation of isotropic materials for plane strain and plane stress conditions}

The stress at a point of a two-dimensional elastic body can be represented by three dependent variables, namely $\sigma_{x x}, \sigma_{y y}$, and $\sigma_{x y}$. With reference to a rectangular coordinate system and in the absence of body forces, these three variables in the case of isotropic materials are governed by two equilibrium equations [29-30].

$$
\begin{aligned}
& \frac{\partial}{\partial x}\left(\sigma_{x x}\right)+\frac{\partial}{\partial y}\left(\sigma_{x y}\right)=0, \\
& \frac{\partial}{\partial y}\left(\sigma_{y y}\right)+\frac{\partial}{\partial x}\left(\sigma_{x y}\right)=0 .
\end{aligned}
$$

The stress-displacement relationships for plane stress and plane strain conditions are as follows [2930]

$$
\begin{aligned}
& \sigma_{x x}(x, y)=\frac{\bar{E}}{1-\bar{v}^{2}}\left[\frac{\partial u_{x}}{\partial x}+\bar{v} \frac{\partial u_{y}}{\partial y}\right] \\
& \sigma_{y y}(x, y)=\frac{\bar{E}}{1-\bar{v}^{2}}\left[\frac{\partial u_{y}}{\partial y}+\bar{v} \frac{\partial u_{x}}{\partial x}\right], \\
& \sigma_{x y}(x, y)=\frac{\bar{E}}{2(1+\bar{v})}\left[\frac{\partial u_{x}}{\partial y}+\frac{\partial u_{y}}{\partial x}\right]
\end{aligned}
$$


where $\bar{E}=E, \bar{v}=v$ for the plane stress condition $\bar{E}=\frac{E}{1-v^{2}}, \bar{v}=\frac{v}{1-v}$ for the plane strain condition,

After substituting the stress-displacement relations as given by Eqs (3.3) to (3.5) in the equilibrium Eqs (3.1) and (3.2), we get the following equilibrium equations for the plane problems of isotropic, elastic bodies of Hookean materials, in terms of displacement components

$$
\begin{aligned}
& \frac{\partial^{2} u_{x}}{\partial x^{2}}+\frac{1-\bar{v}}{2} \frac{\partial^{2} u_{x}}{\partial y^{2}}+\frac{1+\bar{v}}{2} \frac{\partial^{2} u_{y}}{\partial x \partial y}=0 \\
& \frac{\partial^{2} u_{y}}{\partial y^{2}}+\frac{1-\bar{v}}{2} \frac{\partial^{2} u_{y}}{\partial x^{2}}+\frac{1+\bar{v}}{2} \frac{\partial^{2} u_{x}}{\partial x \partial y}=0 .
\end{aligned}
$$

In the displacement potential function formulation, the displacement components are expressed in terms of a potential function $\psi$ of space variables as follows

$$
\begin{aligned}
& u_{x}(x, y)=\frac{\partial^{2} \psi}{\partial x \partial y}, \\
& u_{y}(x, y)=-\frac{1}{1+\bar{v}}\left[2 \frac{\partial^{2} \psi}{\partial x^{2}}+(1-\bar{v}) \frac{\partial^{2} \psi}{\partial y^{2}}\right] .
\end{aligned}
$$

The displacement components as given by Eqs (3.8) and (3.9) are substituted into Eqs (3.6) and (3.7). Equation (3.6) is automatically satisfied, but Eq.(3.7) turns into a governing equation which is as follows

$$
\frac{\partial^{4} \psi}{\partial x^{4}}+2 \frac{\partial^{4} \psi}{\partial x^{2} \partial y^{2}}+\frac{\partial^{4} \psi}{\partial y^{4}}=0
$$

Substituting the expressions as given by Eqs (3.8) and (3.9) into Eqs (3.3) to (3.5) considering the plane strain and the plane stress conditions, one obtains the explicit expressions of three stress components in terms of the potential function, $\psi$

$$
\begin{aligned}
& \sigma_{x x}(x, y)=\frac{\bar{E}}{(1+\bar{v})^{2}}\left[\frac{\partial^{3} \psi}{\partial x^{2} \partial y}-\bar{v} \frac{\partial^{3} \psi}{\partial y^{3}}\right], \\
& \sigma_{y y}(x, y)=-\frac{\bar{E}}{(1+\bar{v})^{2}}\left[(2+\bar{v}) \frac{\partial^{3} \psi}{\partial x^{2} \partial y}+\frac{\partial^{3} \psi}{\partial y^{3}}\right], \\
& \sigma_{x y}(x, y)=\frac{\bar{E}}{(1+\bar{v})^{2}}\left[\bar{v} \frac{\partial^{3} \psi}{\partial x \partial y^{2}}-\frac{\partial^{3} \psi}{\partial x^{3}}\right] .
\end{aligned}
$$




\section{Solution of the present problem}

A stiffened rectangular duralumin plate is considered for the case of plane stress/plane strain conditions. Two different problems are solved changing the boundary conditions in the case of plane strain /plane stress conditions. For the present stiffened plate problems, the potential function, $\psi$ is assumed as

$$
\psi(x, y)=\sum_{m=1}^{\infty} Y_{m} \sin \alpha x
$$

where $Y_{m}$ is a function of $y$ only, and $\alpha=\frac{m \pi}{a}$, then $Y_{m}$ has to satisfy the ordinary differential equation. After substituting the above expressions of $\psi$ to Eq.(3.10), one obtains the following ordinary differential equation

$$
\begin{aligned}
& \frac{d^{4} Y_{m}}{d y^{4}}-2 \alpha^{2} \frac{d^{2} Y_{m}}{d y^{2}}+\alpha^{4} Y_{m}=0 \\
& Y_{m}=A_{m} \cosh \alpha y+B_{m} \alpha y \sinh \alpha y+C_{m} \sinh \alpha y+D_{m} \alpha y \cosh \alpha y
\end{aligned}
$$

where $A_{m}, B_{m}, C_{m}$, and $D_{m}$ are arbitrary constants.

After substituting the expression of $\psi$ as given by Eq.(4.1) into Eqs (3.8), (3.9), (3.11) to (3.13), one obtains the expressions of the displacement and stress components as follows

$$
\begin{aligned}
& u_{x}(x, y)=\alpha \sum_{m=1}^{\alpha} Y_{m}^{\prime} \cos \alpha x, \\
& u_{y}(x, y)=-\frac{1}{1+\bar{v}} \sum_{m=1}^{\alpha}\left[-2 Y_{m} \alpha^{2}+(1-\bar{v}) Y_{m}^{\prime \prime}\right] \sin \alpha x, \\
& \sigma_{x x}(x, y)=-\frac{\bar{E}}{(1+\bar{v})^{2}} \sum_{m=1}^{\infty}\left[Y_{m}^{\prime} \alpha^{2}+\bar{v} Y_{m}^{\prime \prime \prime}\right] \sin \alpha x, \\
& \sigma_{y y}(x, y)=-\frac{\bar{E}}{(1+\bar{v})^{2}} \sum_{m=1}^{\infty}\left[-(2+\bar{v}) \alpha^{2} Y_{m}^{\prime}+Y_{m}^{\prime \prime \prime}\right] \sin \alpha x, \\
& \sigma_{x y}(x, y)=\frac{\bar{E}}{(1+\bar{v})^{2}} \sum_{m=1}^{\infty}\left[\bar{v} Y_{m}^{\prime \prime}+Y_{m} \alpha^{2}\right] \alpha \cos \alpha x .
\end{aligned}
$$

Substituting the derivatives of $Y_{m}$ in the expressions of the displacement and stress components as given in Eqs (4.3) to (4.7), one obtains

$$
u_{x}(x, y)=\alpha^{2} \sum_{m=1}^{\infty}\left[\begin{array}{l}
\left(A_{m}+B_{m}\right) \sinh \alpha y+\left(C_{m}+D_{m}\right) \cosh \alpha y+ \\
B_{m} \alpha y \cosh \alpha y+D_{m} \alpha y \sinh \alpha y
\end{array}\right] \cos \alpha x,
$$




$$
\begin{aligned}
& u_{y}(x, y)=-\frac{1}{1+\bar{v}} \sum_{m=1}^{\alpha}\left[\begin{array}{l}
-2 \alpha^{2}\left(A_{m} \cosh \alpha y+B_{m} \alpha y \sin \alpha y+C_{m} \sinh \alpha y+\right. \\
\left.+D_{m} \alpha y \cosh \alpha y\right)+(1-\bar{v})\left\{\alpha^{2}\left(A_{m}+2 B_{m}\right\} \cosh \alpha y+\right. \\
\left(C_{m}+2 D_{m}\right) \alpha^{2} \sinh \alpha y+B_{m} \alpha^{3} y \sinh \alpha y+D_{m} \alpha^{3} y \cosh \alpha y
\end{array}\right] \times \sin \alpha x,
\end{aligned}
$$

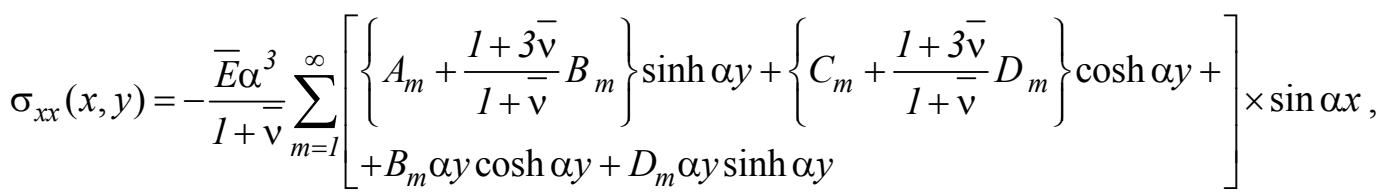

$$
\begin{aligned}
& \sigma_{y y}(x, y)=\frac{\bar{E} \alpha^{3}}{1+\bar{v}} \sum_{m=1}^{\infty}\left[\begin{array}{l}
\left\{A_{m}-t B_{m}\right\} \sinh \alpha y+\left\{C_{m}-t D_{m}\right\} \cosh \alpha y+ \\
+B_{m} \alpha y \cosh \alpha y+D_{m} \alpha y \sinh \alpha y
\end{array}\right] \times \sin \alpha x, \\
& \sigma_{x y}(x, y)=\frac{\bar{E} \alpha^{3}}{1+\bar{v}} \sum_{m=1}^{\infty}\left[\begin{array}{l}
\left\{A_{m}+(1-t) B_{m}\right\} \cosh \alpha y+\left\{C_{m}+(1-t) D_{m}\right\} \sinh \alpha y+ \\
+B_{m} \alpha y \sinh \alpha y+D_{m} \alpha y \cosh \alpha y
\end{array}\right] \times \cos \alpha x
\end{aligned}
$$

where $\quad t=\frac{1-\bar{v}}{1+\bar{v}}$.

\subsection{Applications of the boundary conditions:}

\subsubsection{The boundary conditions of the problem as shown in Fig.1 are as follows}

The boundary conditions at the supporting edge $\mathrm{AB}$ are

$$
u_{x}(x, 0)=0 ; \quad u_{y}(x, 0)=0 \text {. }
$$

The boundary conditions at the top vertical edge, $\mathrm{AD}$ are

$$
u_{y}(0, y)=0 ; \quad \sigma_{x x}(0, y)=0 .
$$

The boundary conditions at the bottom vertical edge, $\mathrm{BC}$ are

$$
u_{y}(a, y)=0 ; \quad \sigma_{x x}(a, y)=0 .
$$

The boundary conditions at the loading edge, $\mathrm{CD}$

$$
\begin{aligned}
& \sigma_{y y}(x, b)=P=E_{0}+\sum_{m=1}^{\infty} E_{m} \sin \alpha x, \\
& u_{x}(x, b)=0, \\
& E_{0}=0 ; \quad E_{m}=\frac{2}{a} \int_{0}^{a} p \sin \alpha x d y=\frac{4 p}{m \pi} \quad \text { when } \quad m=1,3,5 \ldots \ldots
\end{aligned}
$$




\subsubsection{The boundary conditions of the problem as shown in Fig.2 are as follows}

The boundary conditions at the edge EF are

$$
\begin{aligned}
& \sigma_{y y}(x, 0)=P=E_{0}^{\prime}+\sum_{m=1}^{\infty} E_{m} \sin \alpha x, \\
& E_{0}=0 ; \quad E_{m}=\frac{2}{a} \int_{0}^{a} p \sin \alpha x d y=\frac{4 p}{m \pi} \quad \text { when } m=1,3,5 \ldots \ldots . \\
& u_{x}(x, 0)=0 .
\end{aligned}
$$

The boundary conditions at the edge EH are

$$
\begin{aligned}
& u_{y}(0, y)=0, \\
& \sigma_{x x}(0, y)=0 .
\end{aligned}
$$

The boundary conditions at the edge GH are

$$
\begin{aligned}
& u_{x}(x, b)=0, \\
& \sigma_{y y}(x, b)=P=E_{0}^{\prime \prime}+\sum_{m=1}^{\infty} E_{m} \sin \alpha x \\
& E_{0}^{\prime \prime}=0 ; \quad E_{m}=\frac{2}{a} \int_{0}^{a} p \sin \alpha x d y=\frac{4 p}{m \pi} \quad \text { when } \quad m=1,3,5 \ldots \ldots
\end{aligned}
$$

The boundary conditions at the edge FG are

$$
\begin{aligned}
& u_{y}(a, y)=0, \\
& \sigma_{x x}(a, y)=0 .
\end{aligned}
$$

Firstly, substituting the boundary conditions of the relevant problems associated with Fig.1 (Eq.(4.13) to Eq.(4.17)) and Fig.2 (Eq.(4.18) to Eq.(4.25) into the general expressions of the displacement and stress components as given by Eqs (4.8) to (4.12), one obtains the four unknown coefficients $A_{m}, B_{m}, C_{m}$, and $D_{m}$.After substituting the value of $A_{m}, B_{m}, C_{m}$, and $D_{m}$ into Eqs (4.8) to (4.12), one obtains the displacement and stress components throughout the plate for two different problems.

The following table shows the expressions of the relevant unknown constants $A_{m}, B_{m}, C_{m}$ and $D_{m}$ for two different problems as shown in Fig.1 and Fig.2. 


\begin{tabular}{|c|c|c|}
\hline $\begin{array}{l}\text { Unknown } \\
\text { constants }\end{array}$ & Problem 1 as shown in Fig.1 & Pproblem 2 as shown in Fig.2 \\
\hline$A_{m}$ & $2 t B_{m}$ & {$\left[E_{m}(1+\bar{v}) n\right] /\left\{\bar{E} \alpha^{3}(t+1) \sinh ^{2}(c)\right\}$} \\
\hline$B_{m}$ & $\left\{E_{m}(1+\bar{v}) b \sinh (b)\right\} / n$ & {$\left[E_{m}(1+\bar{v})\{\cosh (c)-1\}\right] /\left\{\bar{E} \alpha^{3}(t+1) \sinh (c)\right\}$} \\
\hline$C_{m}$ & $\left\{E_{m}(2 t+1) \sinh (b)+b \cosh (b)(1+\bar{v})\right\} / n$ & $\left\{E_{m}(1+\bar{v})\right\} /\left\{\bar{E} \alpha^{3}(t+1)\right\}$ \\
\hline$D_{m}$ & $-C_{m}$ & $-\left\{E_{m}(1+\bar{v})\right\} /\left\{\bar{E} \alpha^{3}(t+1)\right\}$ \\
\hline
\end{tabular}

where $n=\{(1+t)(2 t+1) \sinh (b) \cosh (b)+b\} \bar{E} \alpha^{3}$.

\section{Results and discussion}

Figure 1.1 shows the deformed shapes of the plate as observed in Fig. 1 in the case of plane stress and plane strain conditions. The deformations of two edges such as $\mathrm{AD}$ and $\mathrm{BC}$ along the $y$ direction and the deformation of the edge $\mathrm{CD}$ along the $x$ direction and the deformations of the supporting edge $\mathrm{AB}$ are zero as shown in Fig.1.1 which are in good agreement to the physical characteristics of the problem. According to the loading configuration as observed in Fig.1, the deformation of edges $\mathrm{AD}$ and $\mathrm{BC}$ occurs along the $x$ direction except two edges $\mathrm{AB}$ and $\mathrm{CD}$ for both of the cases and the deformations of the plate shown in Fig.1 in both of the cases differ. Figures 1.2 and 1.3 illustrate the parabolically varied axial stress and antisymmetrically varied shear stress components at the section $y / b=0$ of the plate as a function of $x / a$ for the case of plane stress and plane strain conditions. For plane strain conditions, the axial and shear stress components are higher than those of the plane stress condition because the stiffness of the plate in the case of the plane strain condition is higher than that of plane stress. Figures 1.4 and 1.5 illustrate the lateral and axial displacement component at the section $y / b=0.5$ of the plate in the case of plane stress and plane strain conditions. At the section, $y / b=0.5$, the lateral displacement for plane strain condition is higher than that of the plane stress condition because Poisson's ratio (lateral strain/axial strain) for the case of the plane strain plate problem will be higher than that of the plane stress plate problem. According to the theoretical formulation of plane stress and plane strain problems mentioned above in the theoretical sections the lateral displacement of any section of the plate in the case of the plane strain problem will be higher than that of the plane stress problem. No significant effect of plane strain and plane stress conditions on the axial displacement component at the section, $y / b=0.5$ as observed in Fig.1.5. Figures 1.6, 1.7 and 1.8 illustrate the lateral, axial and the shear stress component at the section, $y / b=0.5$, of the plate for the case of plane stress and plane strain conditions. The lateral stress component at the section, $y / b=0.5$, for the plane stress condition is higher than that of plane strain condition as observed in Fig.1.6. But the opposite phenomenon is observed in the case of the axial stress component at the section, $y / b=0.5$, as observed in Fig. 1.7 because the stiffness of the plate along its lateral direction in the case of plane strain condition is lower than that of the plane stress condition. There are no effects on the plane stress and plane strain conditions for the shear stress component at the section, $y / b=0.5$ as shown in Fig.1.8. Figure 1.9 illustrates the comparison of the axial displacement component at the loading section for the plane stress and plane strain conditions. For the plane strain condition, the axial displacement component at the loading section is lower than that of the plane stress condition because the plane strain plate has a higher stiffness along the axial direction than that of its lateral direction. Figures 1.10 and 1.11 show the effect of plane stress and plane strain conditions on the lateral and shear stress component at the loading section, $y / b=1$. The lateral stress at the section, $y / b=1$ for the plane strain condition is higher than that for the plane stress condition because the stiffness of the plane strain plate is higher than that of the plane stress plate problem. But the opposite phenomenon is observed for the shear stress component at the section, $y / b=1$. 
Figure 1.12 illustrates the normalized normal stress component, $\sigma_{z z} / P$ at different sections of the plate. At the loading section, the normal stress, $\sigma_{z z}$ is the highest and towards the supporting edge, the normal stress, $\sigma_{z z}$ gradually decreases. At the two stiffened edges, the normal stress vanishes.

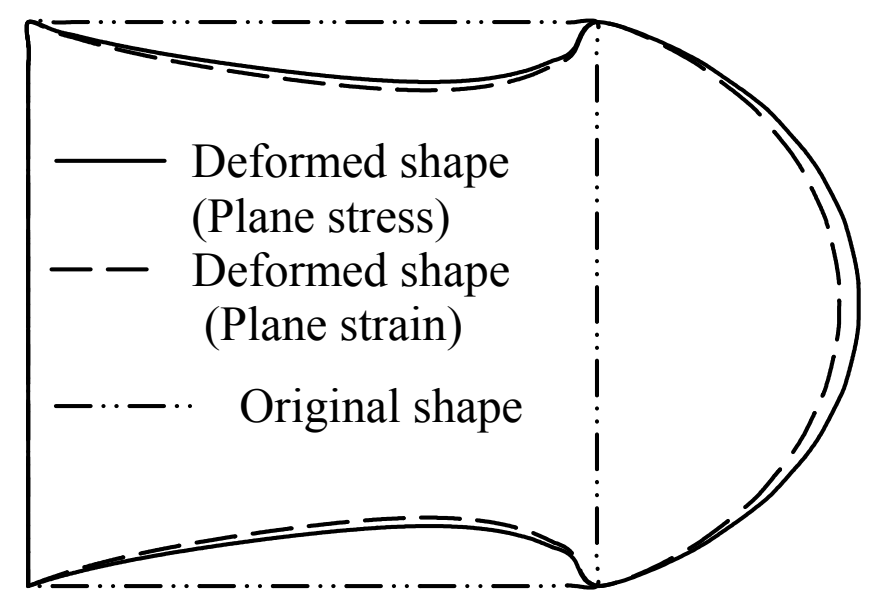

Fig.1.1. Deformed shape of the plate subjected to a uniform tension at its right lateral edge (both the displacements are magnified by 1000 time.

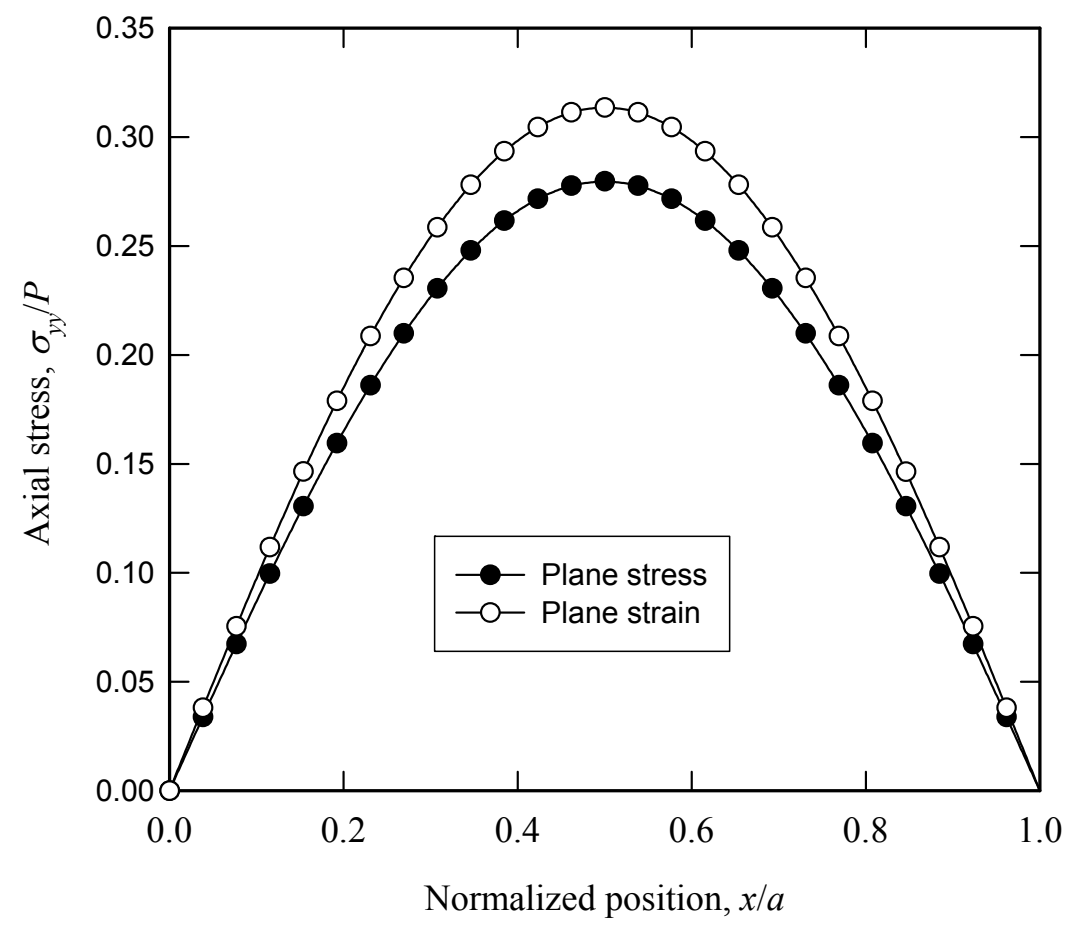

Fig.1.2. Comparison of the axial stress component, $\sigma_{y y}$ at the section, $y / b=0$ obtained by the plane stress and the plane strain conditions. 


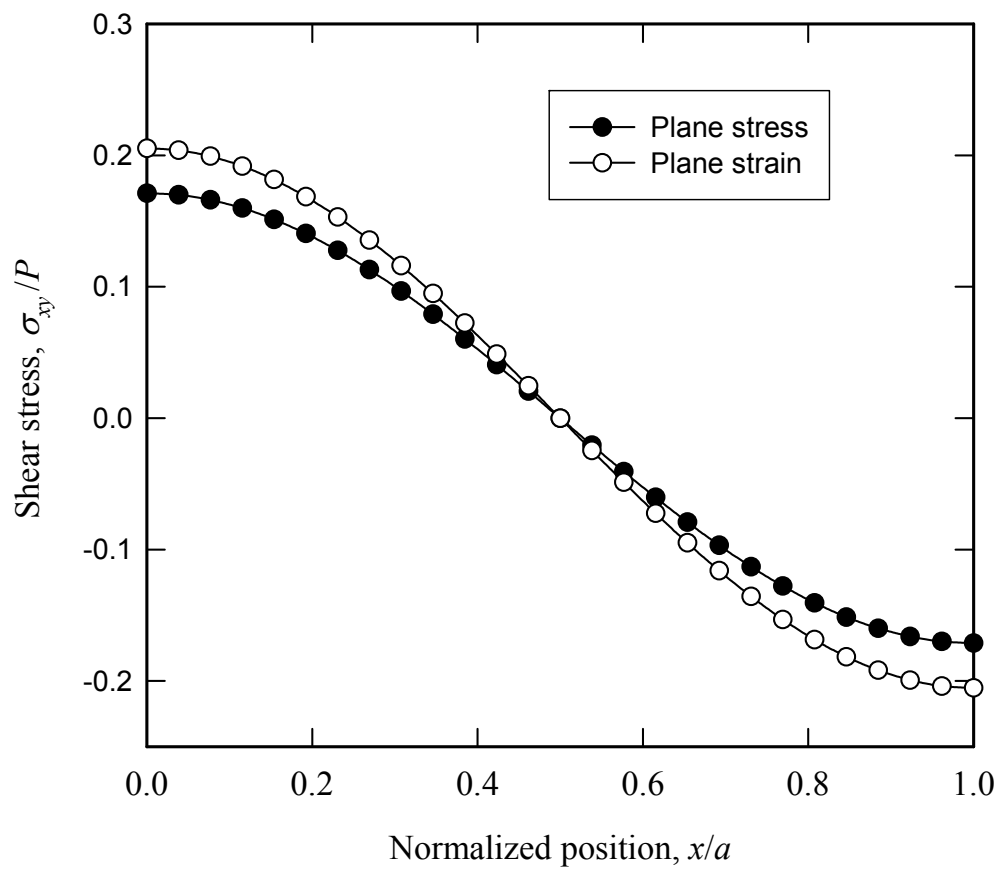

Fig.1.2. Comparison of the shear stress component, $\sigma_{x y}$ at the section, $y / b=0$ obtained by the plane stress and the plane strain conditions.

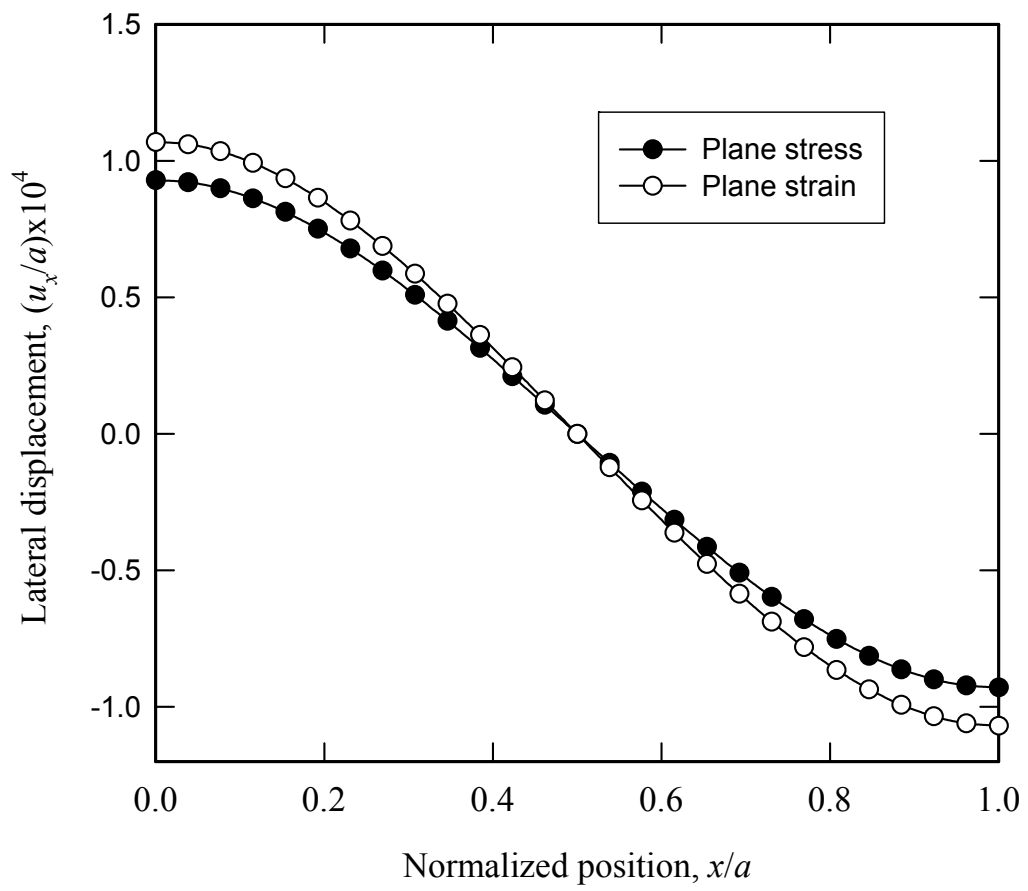

Fig.1.4. Comparison of the lateral displacement component at the section, $y / b=0.5$ obtained by the plane stress and the plane strain conditions. 


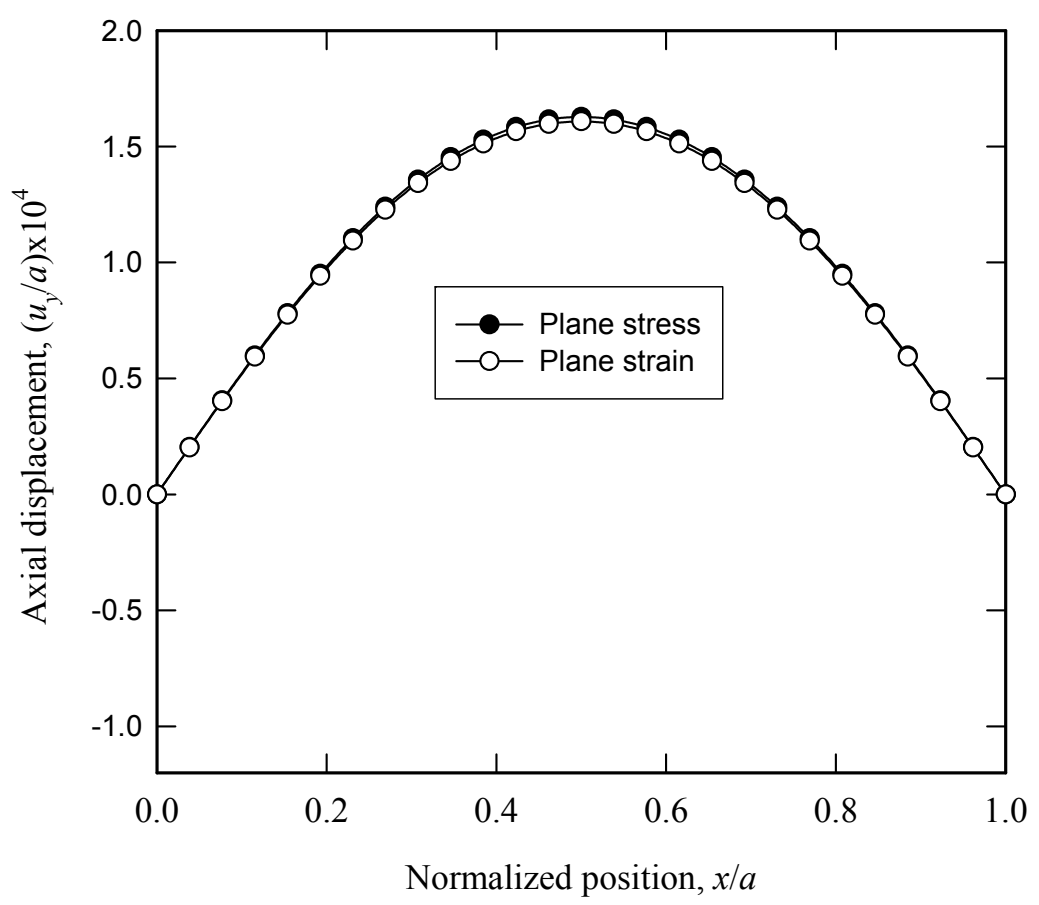

Fig.1.5. Comparison of the axial displacement component at the section, $y / b=0.5$ obtained by the plane stress and the plane strain conditions.

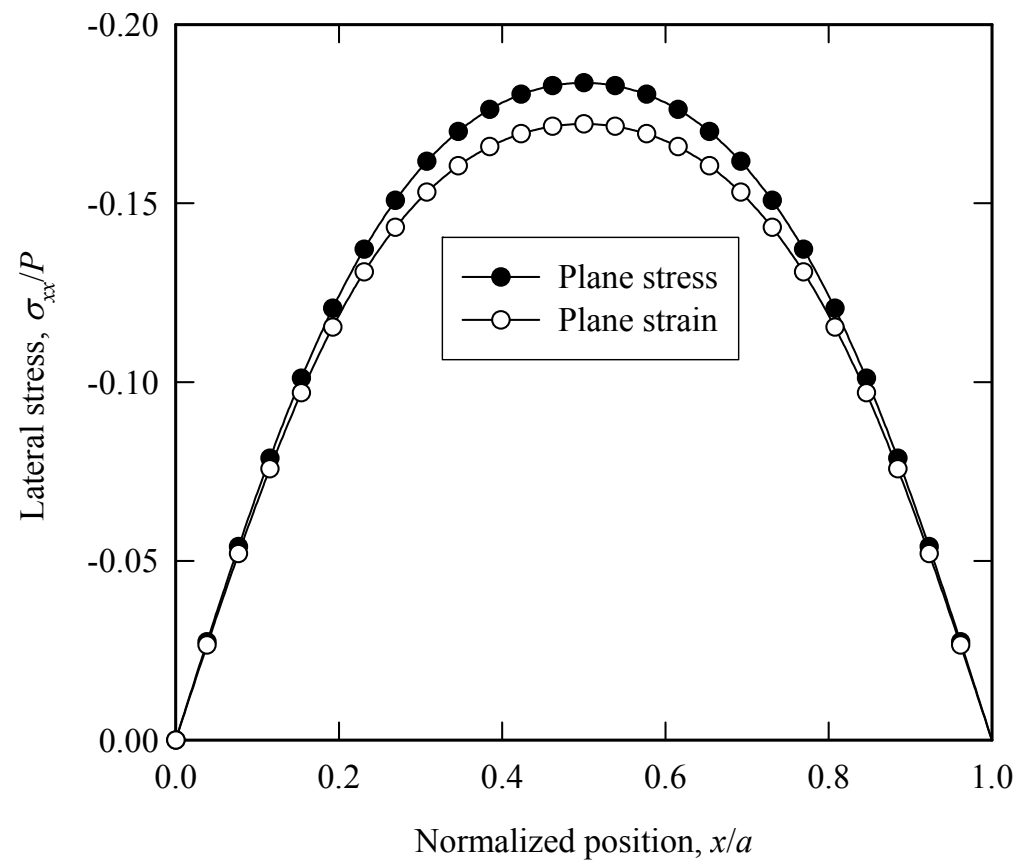

Fig.1.6. Comparison of the lateral stress component $\sigma_{x x}$ at the section, $y / b=0.5$ obtained by the plane stress and the plane strain conditions. 


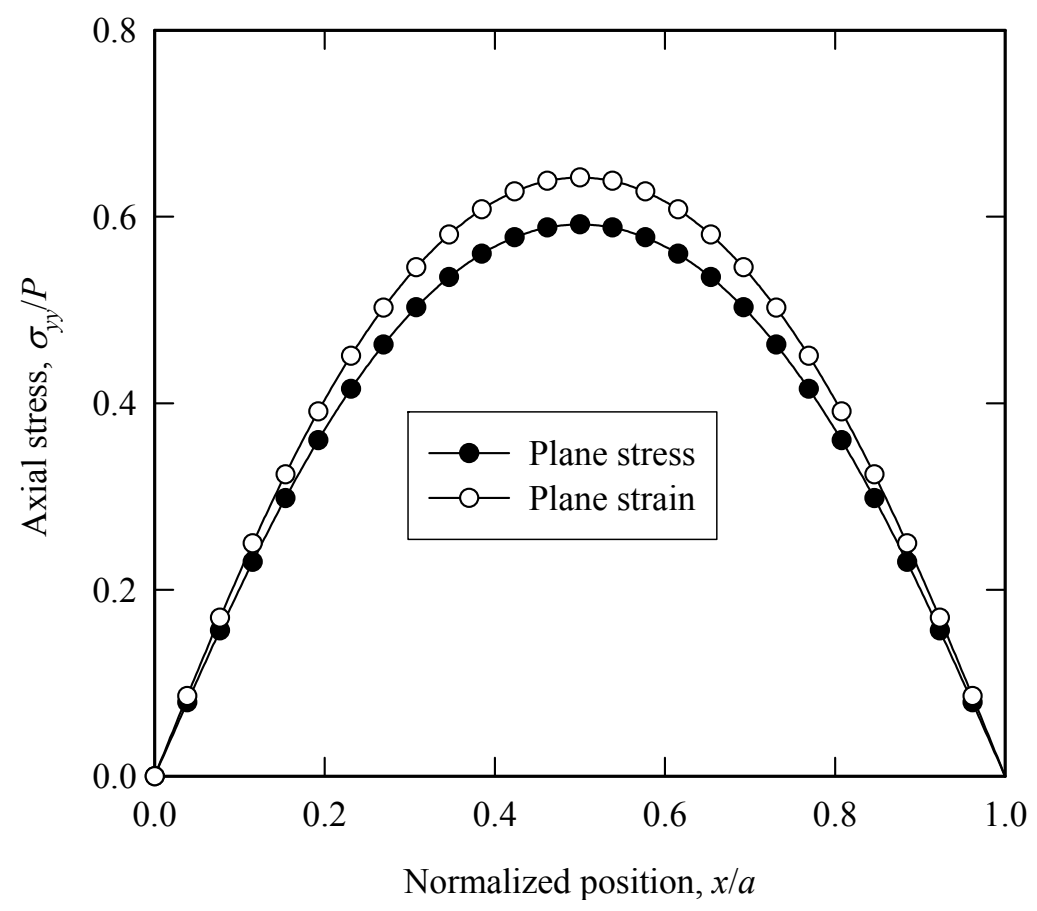

Fig.1.7. Comparison of the axial stress component $\sigma_{y y}$ at the section, $y / b=0.5$ obtained by the plane stress and the plane strain conditions.

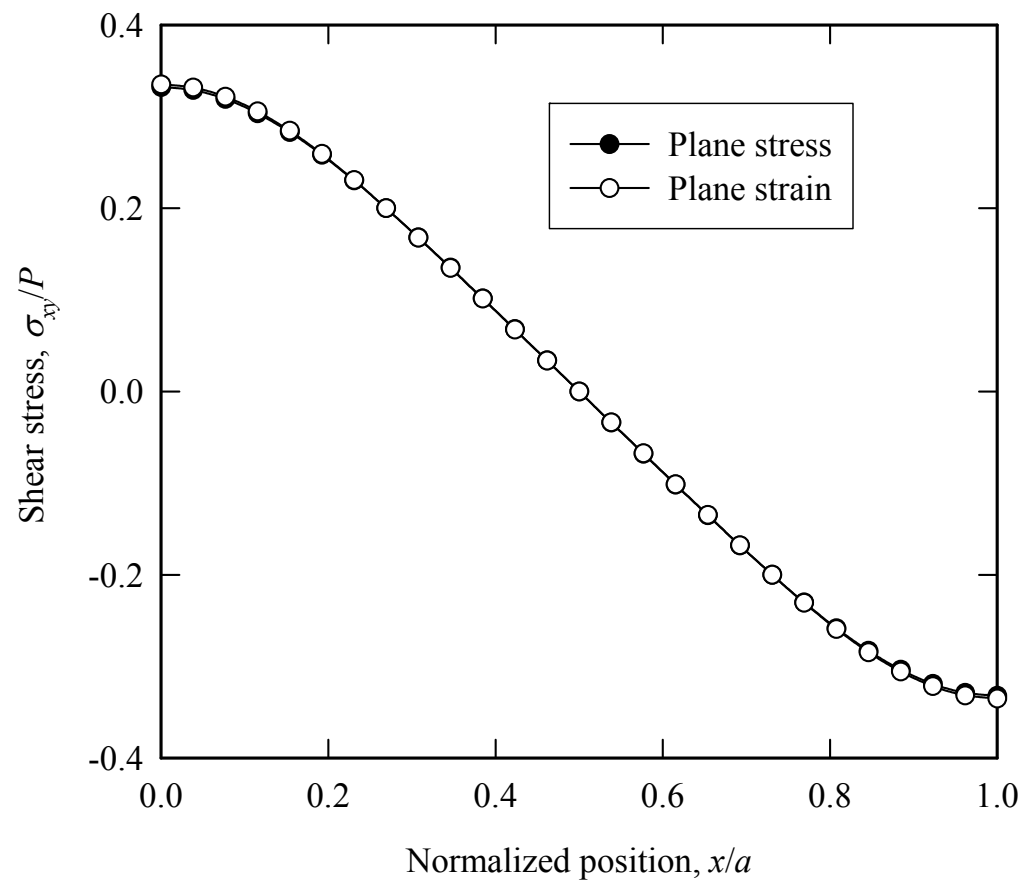

Fig.1.8. Comparison of the shear stress component $\sigma_{x y}$ at the section, $y / b=0.5$ obtained by the plane stress and the plane strain conditions. 


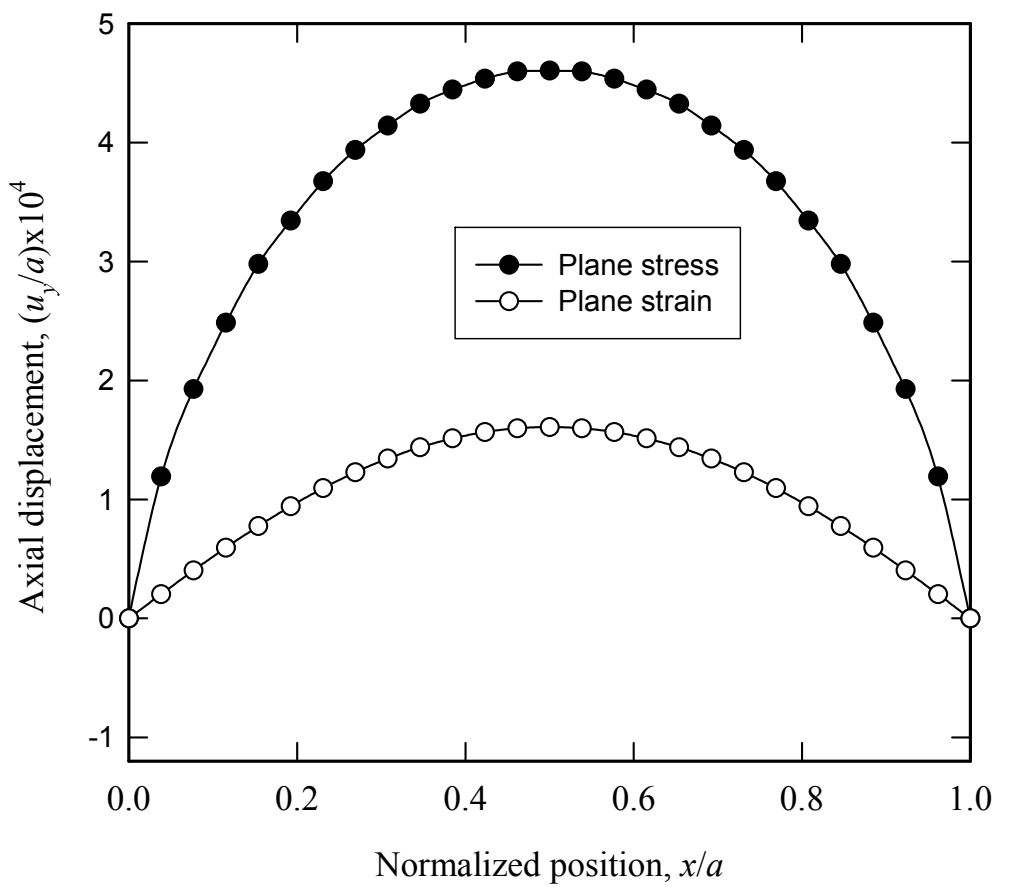

Fig.1.9. Comparison of the axial displacement component at the section, $y / b=1.0$ obtained by the plane stress and the plane strain conditions.

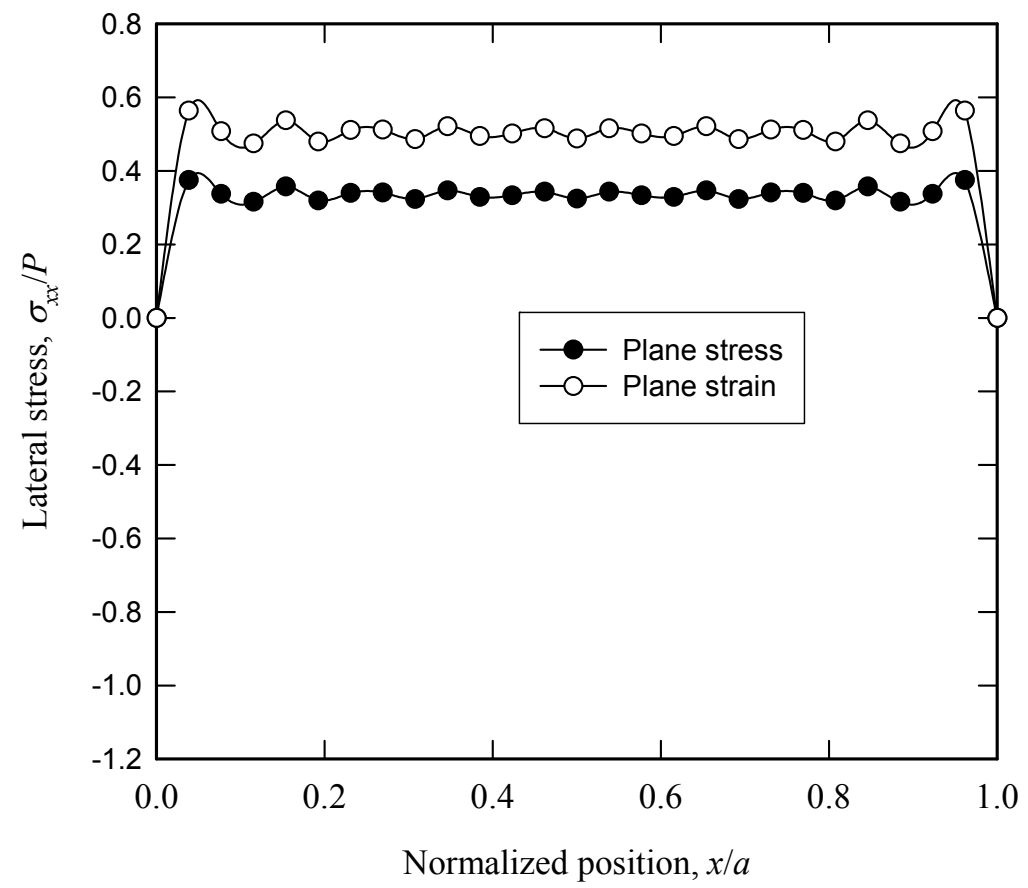

Fig.1.10. Comparison of the lateral stress component at the section, $y / b=1$ obtained by the plane stress and the plane strain conditions. 


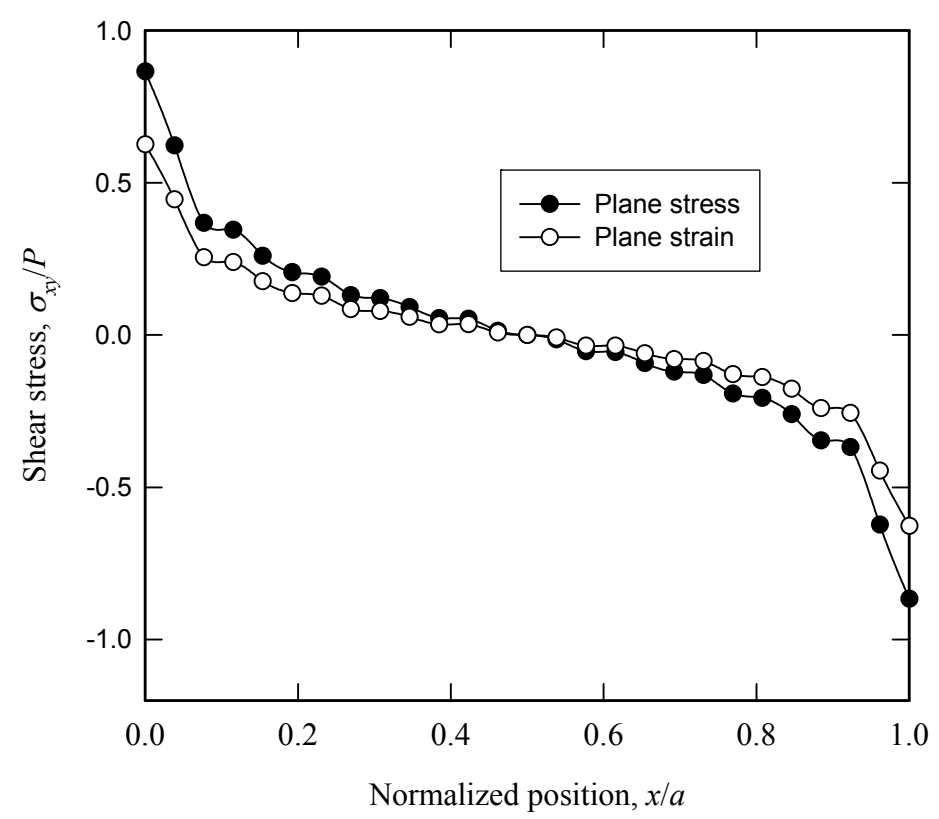

Fig.1.11. Comparison of the shear stress component at the section, $y / b=1$ obtained by the plane stress and the plane strain conditions.

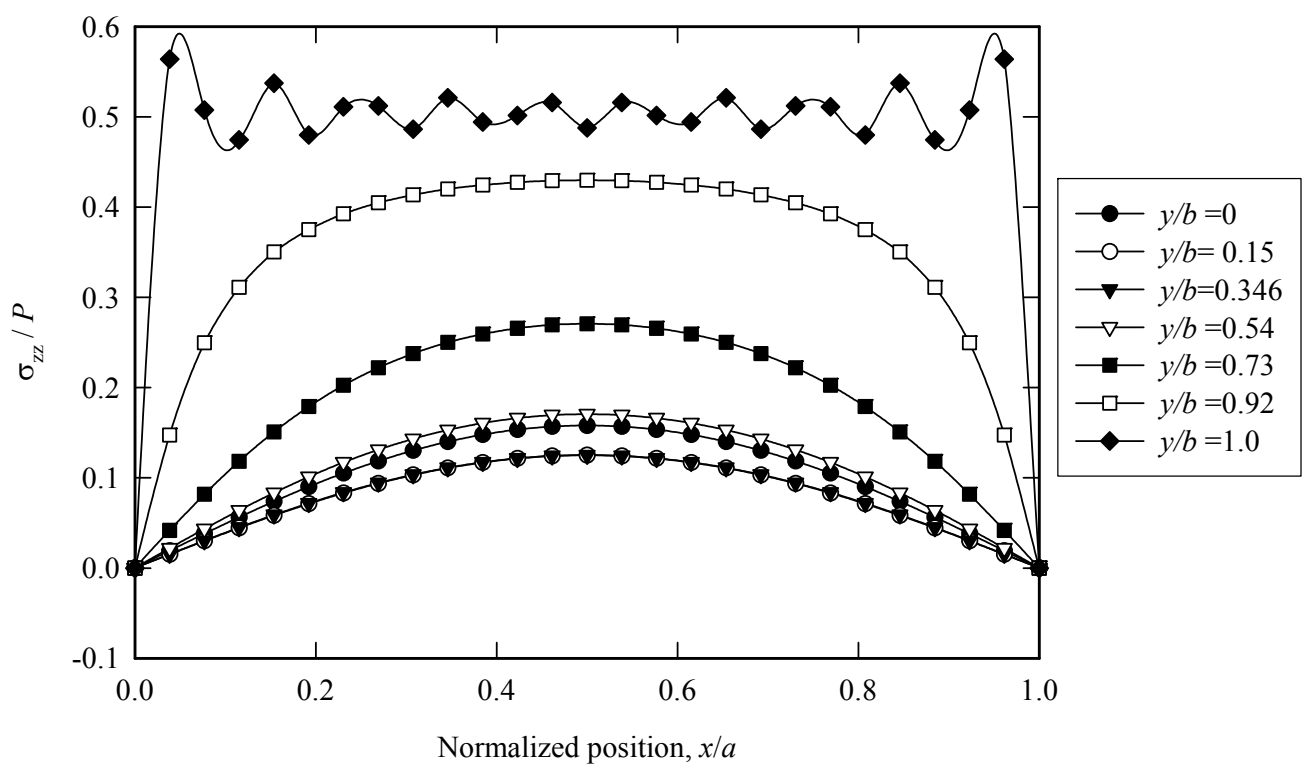

Fig.1.12. Distribution of the normalized normal stress component, $\sigma_{z z} / P$ at different sections of the plate.

From the deformation pattern of the plate as shown in Fig.2.1, the contraction of the plate along the middle section of the plate for plane strain and plane stress conditions is the highest and gradually decreases towards the end points E, F, G, and $\mathrm{H}$ respectively which satisfies the physical characteristics of the problems. The contraction along the $x$ direction of the plate for the plane strain condition is higher than that of the plane stress condition. The expansions at both ends of the plate for the case of the plane stress condition is higher than that of the plane strain condition because the stiffness of the plate and its equivalent Poisson's ratio for the case of plane strain plate is higher than that of plane stress plate as shown in Figs 2.1 and 2.2. At the loading section of the plate, the lateral stress is higher for the plane strain condition than for the plane stress condition because the stiffness of the plate for the plane strain condition is higher than that of the plane stress condition as shown in 
Fig.2.3. But for the shear stress component as observed in Fig.2.4, the opposite phenomenon is observed. Figure 2.5 shows a comparison between the axial displacement component at the section, $x / a=0$ as a function of $y / b$ for the plane stress and plane strain conditions. The axial displacement component of the plane strain condition is higher than that for the plane stress condition. Figure 2.6 illustrates a comparison between the shear stress component at the section, $x / a=0$ as a function of $y / b$ for the plane stress and plane strain conditions. Over the range $0.4 \leq y / b \leq 0.6$ of the section, $x / a=0$, the difference between the shear stress component for both of the cases is zero, but over the rest of the range $0 \leq y / b \leq 0.4$ and $0.6 \leq y / b \leq 1$, the deviation between the shear stress component for both of the cases increases towards the left and right lateral edges of the plate and the shear stress of the plane stress condition is higher than that of the plane strain condition. Figure 2.7 illustrates the normalized normal stress component, $\sigma_{z z}$ at different sections of the plate. Along the middle region of the plate, $y / b=0.5$ except the loading edge, the distribution of the normal stress, $\sigma_{z z}$ is parabolic, and away from the middle section, the normal stress $\sigma_{z z}$ gradually increases.

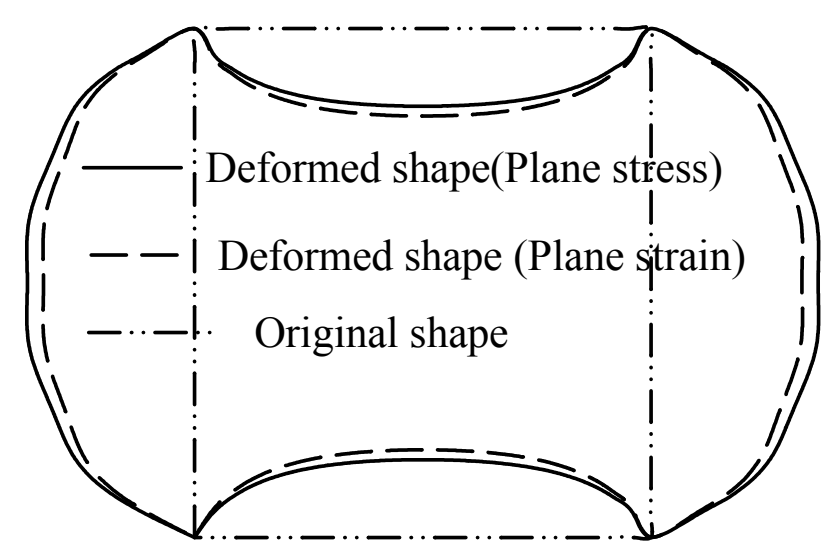

Fig.2.1. Deformed shape of the plate subjected to a uniform tension at its two opposite edges (both the displacements are magnified by 1000 times.

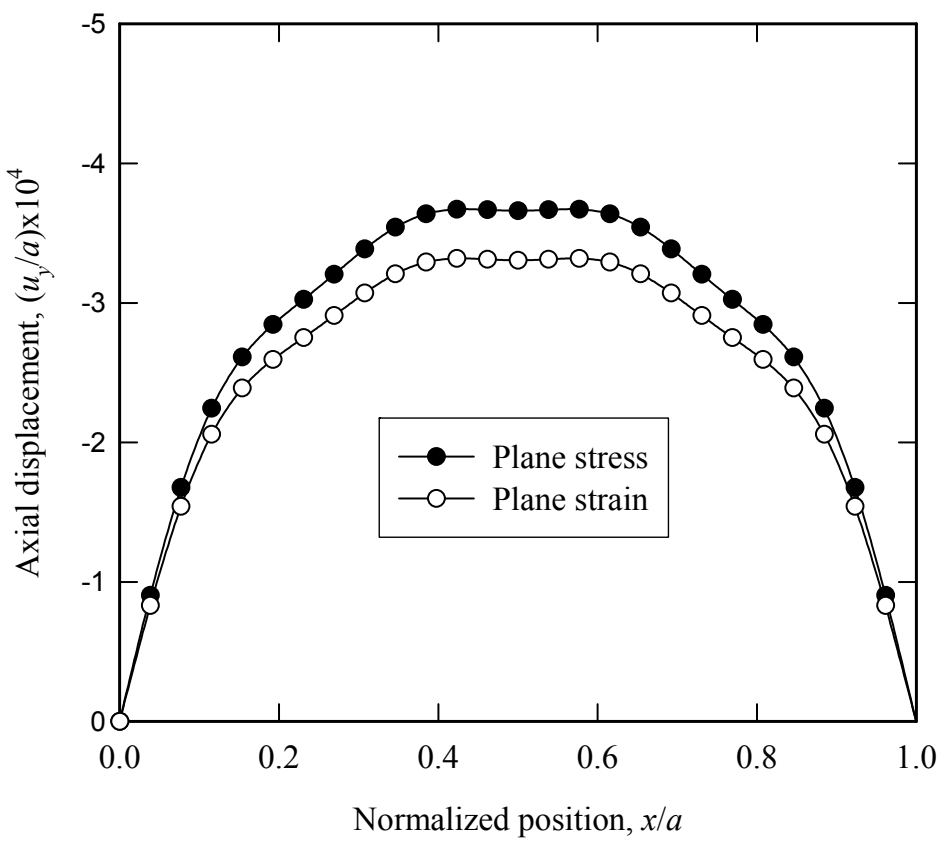

Fig.2.2. Comparison of the axial displacement component at the section, $y / b=0$ obtained by the plane stress and the plane strain conditions. 


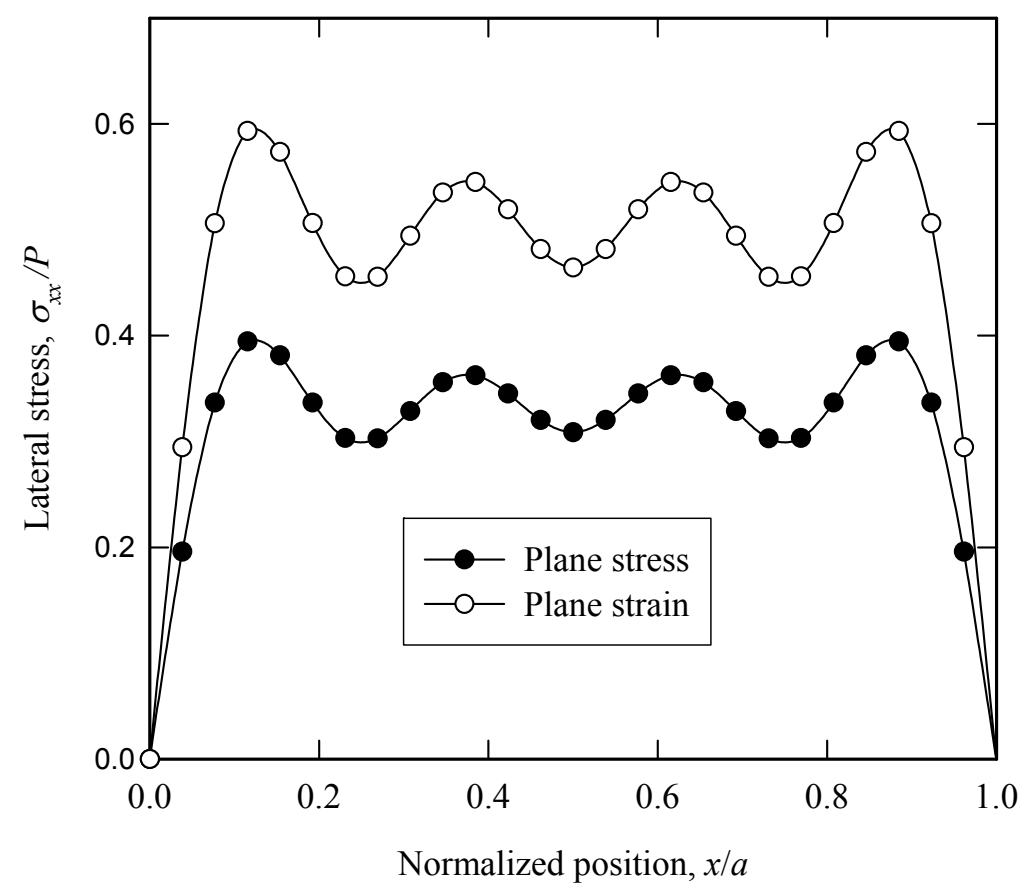

Fig.2.3. Comparison of the lateral stress component at the section, $y / b=0$ obtained by the plane stress and the plane strain conditions.

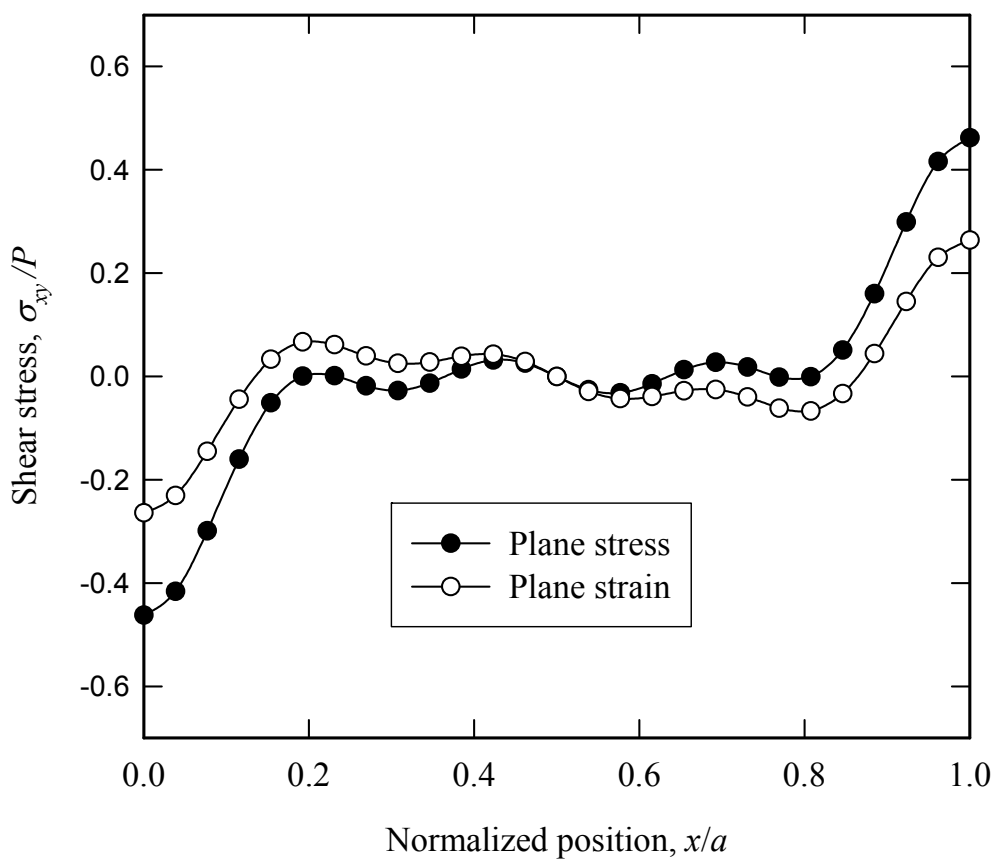

Fig.2.4. Comparison of the shear stress component at the section, $y / b=0$ obtained by the plane stress and the plane strain conditions. 


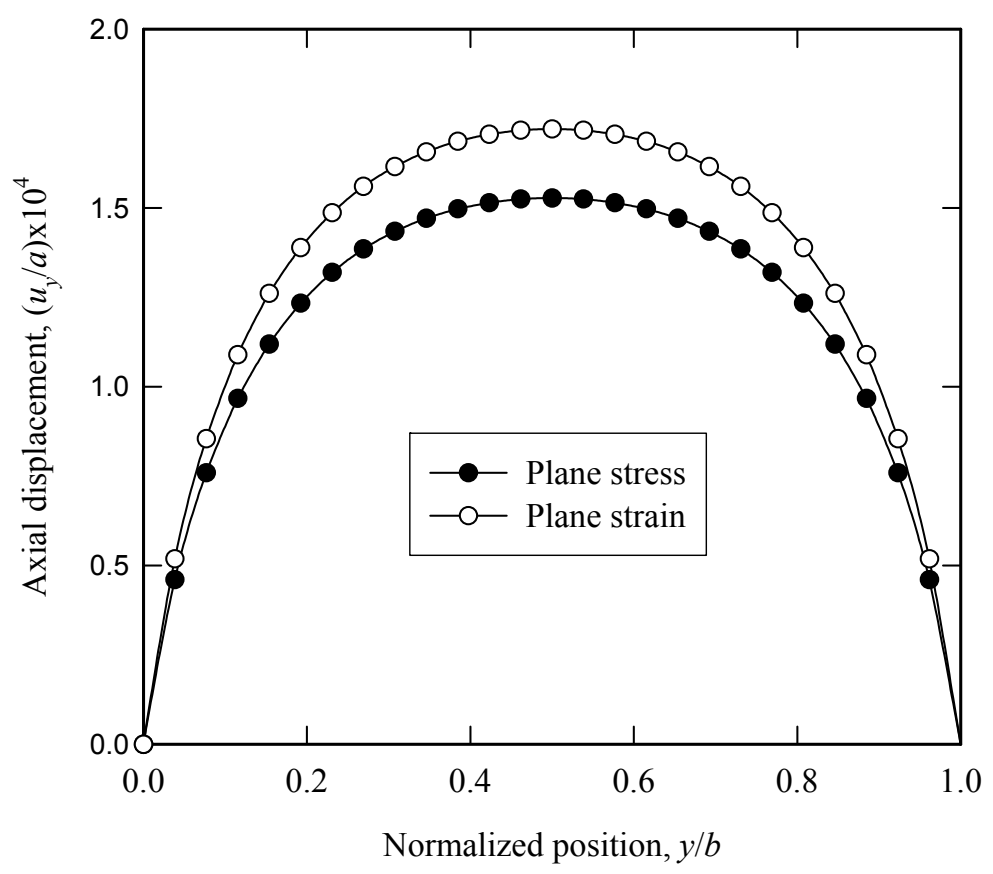

Fig.2.5. Comparison of the axial displacement component at the section, $x / a=0$ obtained by the plane stress and the plane strain conditions.

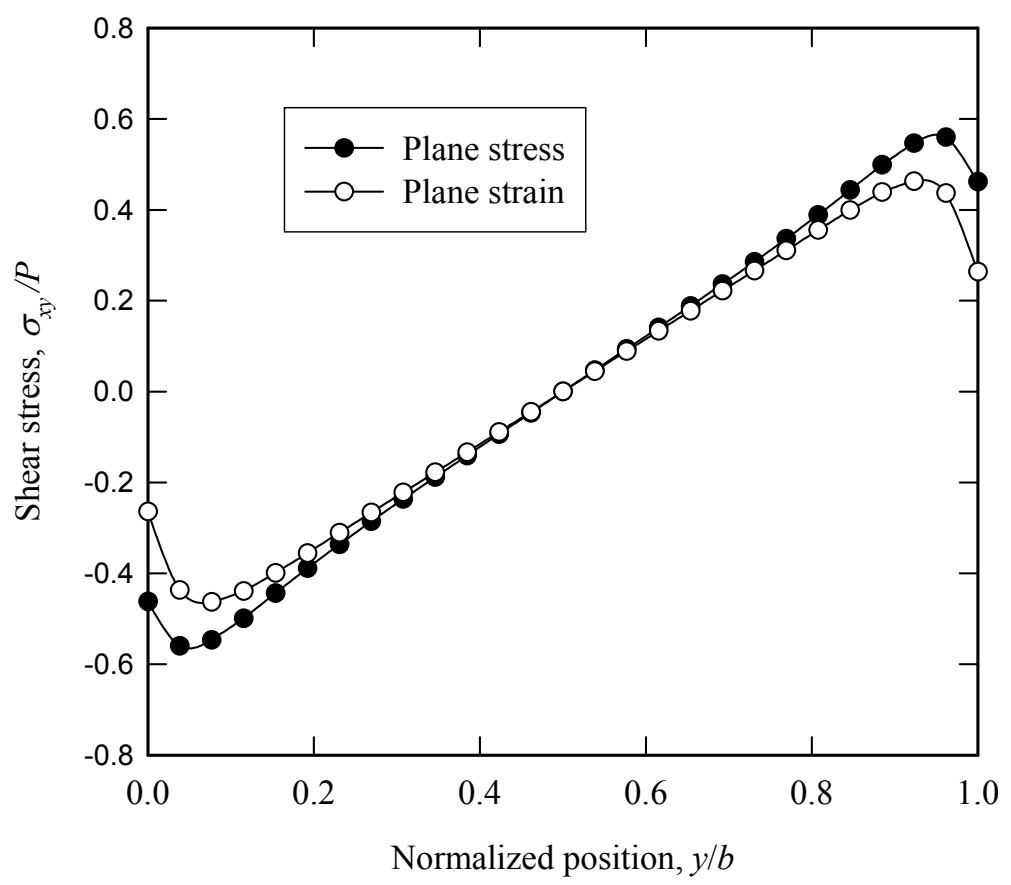

Fig.2.6. Comparison of the axial displacement component at the section, $x / a=0$ obtained by the plane stress and the plane strain conditions. 


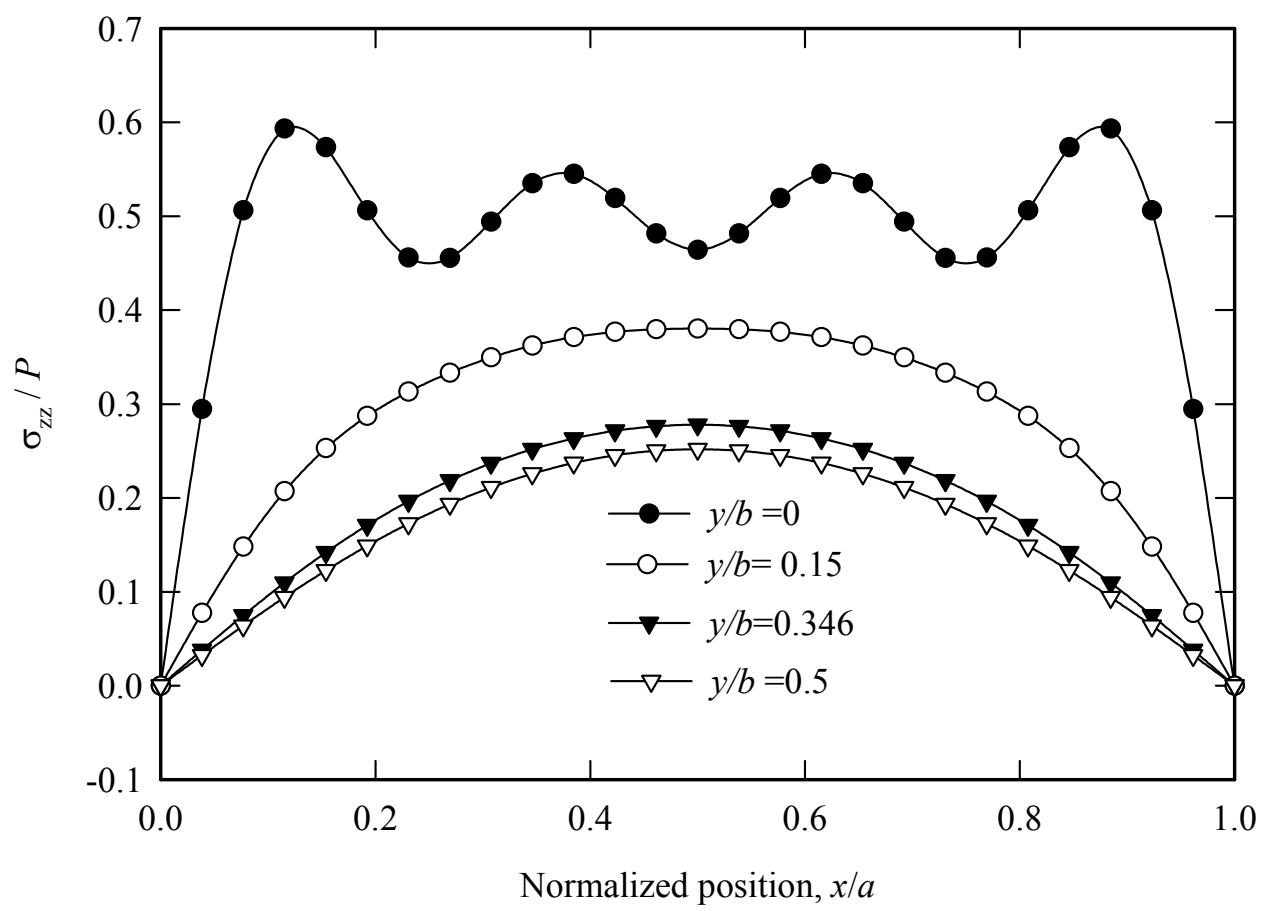

Fig.2.7. Distribution of the normalized normal stress component, $\sigma_{z z} / P$ at different sections of the plate.

To validate the present study, we solve the problem shown in Fig. 1 for the case of the plane stress condition using finite element method and analytical approach. Different components of stress and displacements for both of the methods are compared to each other. Figures $3 \mathrm{a}$ and $3 \mathrm{~b}$ illustrate a comparative study of the axial displacement component, $u_{\mathrm{x}} / a$ and the lateral displacement component $u_{y} / a$ at the section $y / b=0.5$ as a function of $x / a$ obtained by the present analytical approach and finite element method. From Fig.3a it follows that the axial displacements obtained by both of the methods coincide. But Fig.3b there are some discrepancies between the lateral displacements obtained from the present study and finite element method. Figures $4 \mathrm{a}, 4 \mathrm{~b}$ and $4 \mathrm{c}$ show a comparative study of the axial, lateral and shear stress components of the section, $y / b=0.5$ as a function of $x / a$ obtained by the finite element method and the present study. The shear stress obtained from the present study coincides with that of the finite element method as shown in Fig.4c. But there are some discrepancies of the lateral and axial stresses at the section $y / b=0.5$ obtained from the present study with those of the finite element method. 


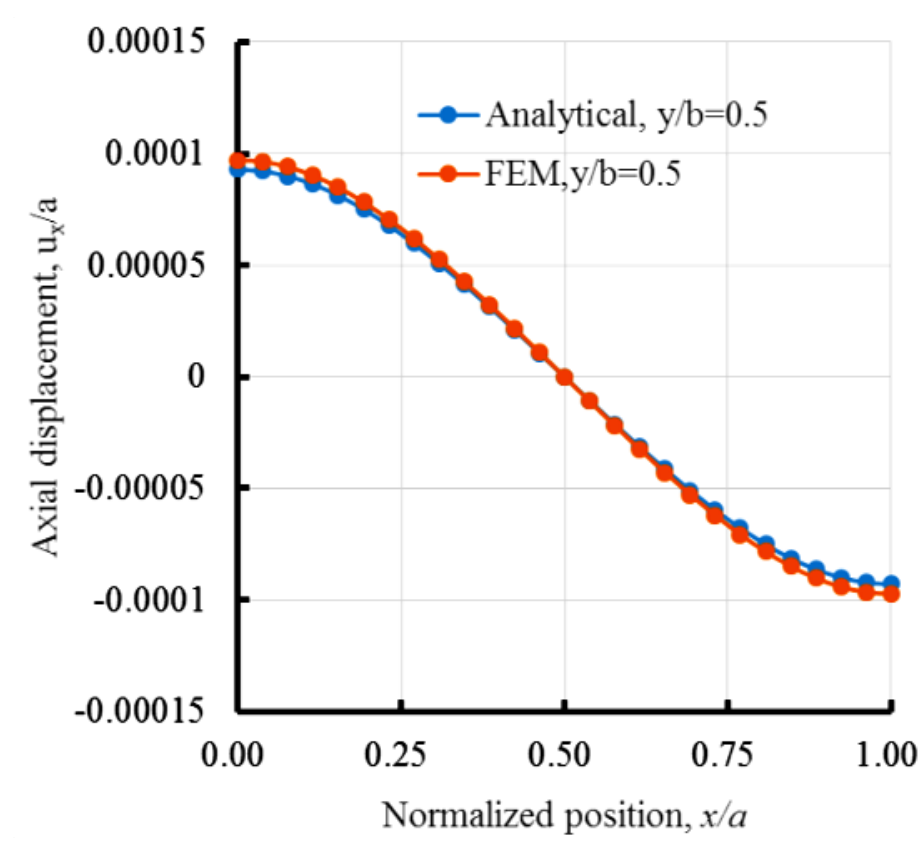

3(a)

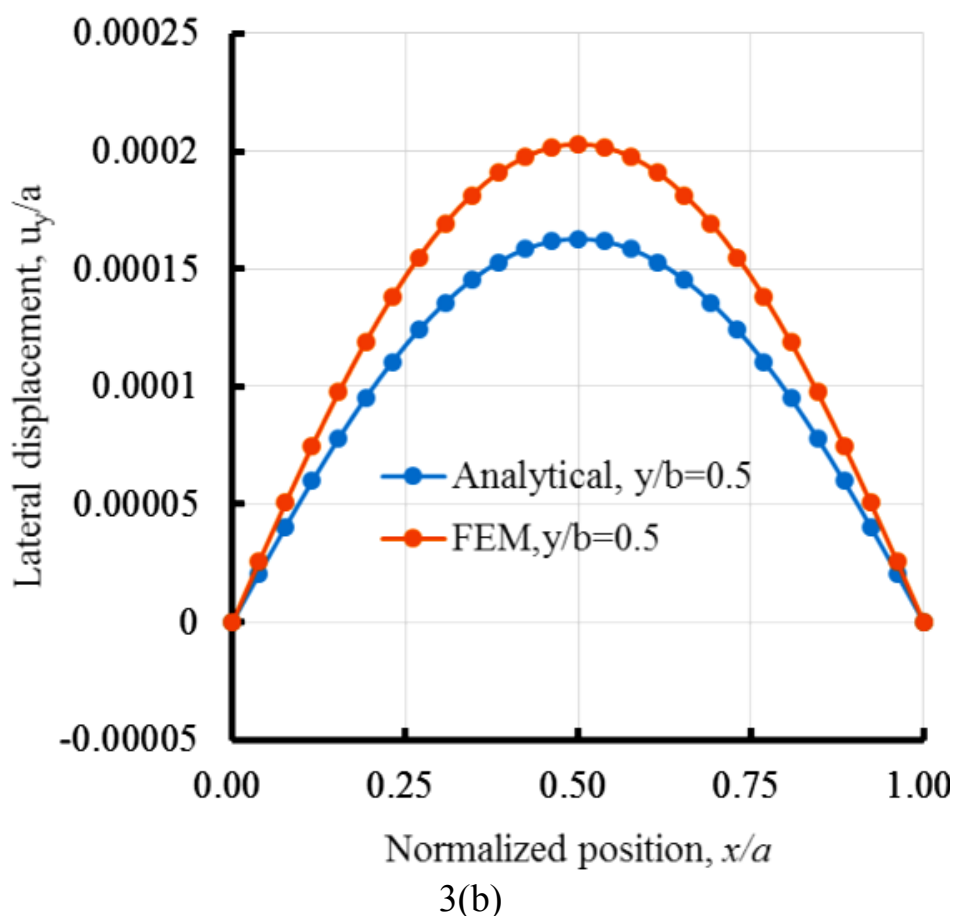

Fig.3. Comparison of the displacement components: (a) axial displacement; (b) lateral displacement at the section $y / b=0.5$ of the duralumin plate considering plane stress condition shown in Fig. 1 as a function of $x / a$ obtained by the analytical and finite element method. 


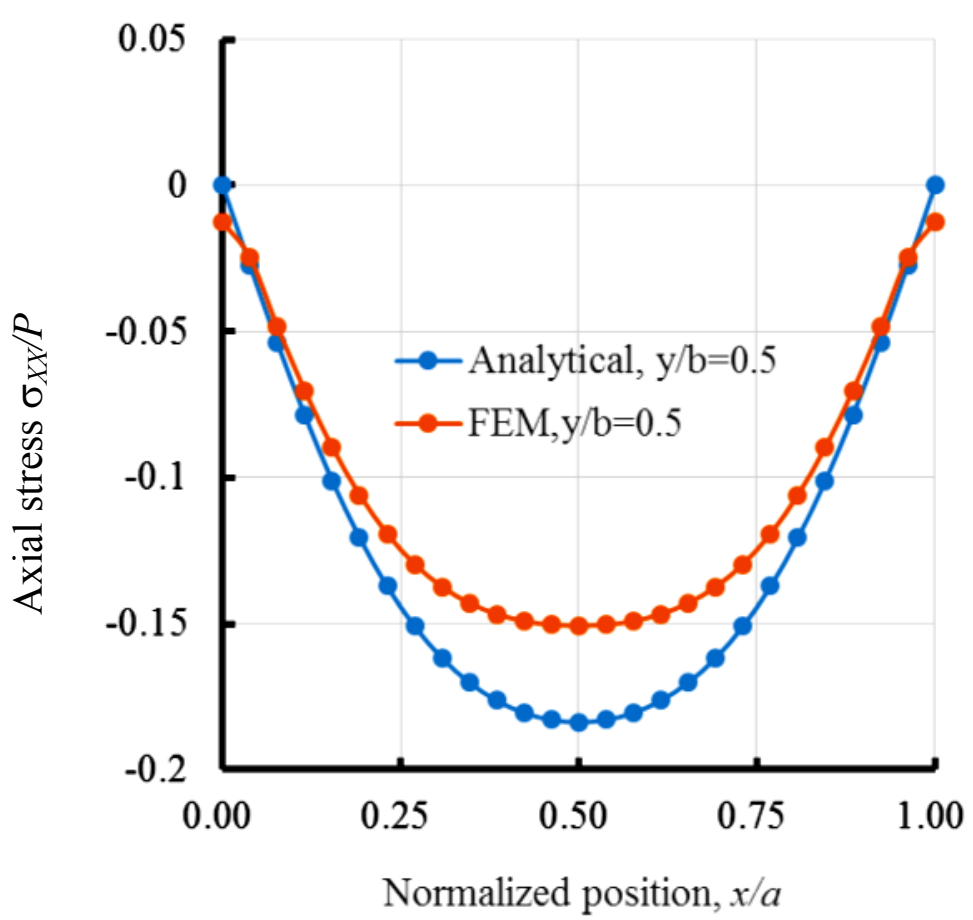

4(a)

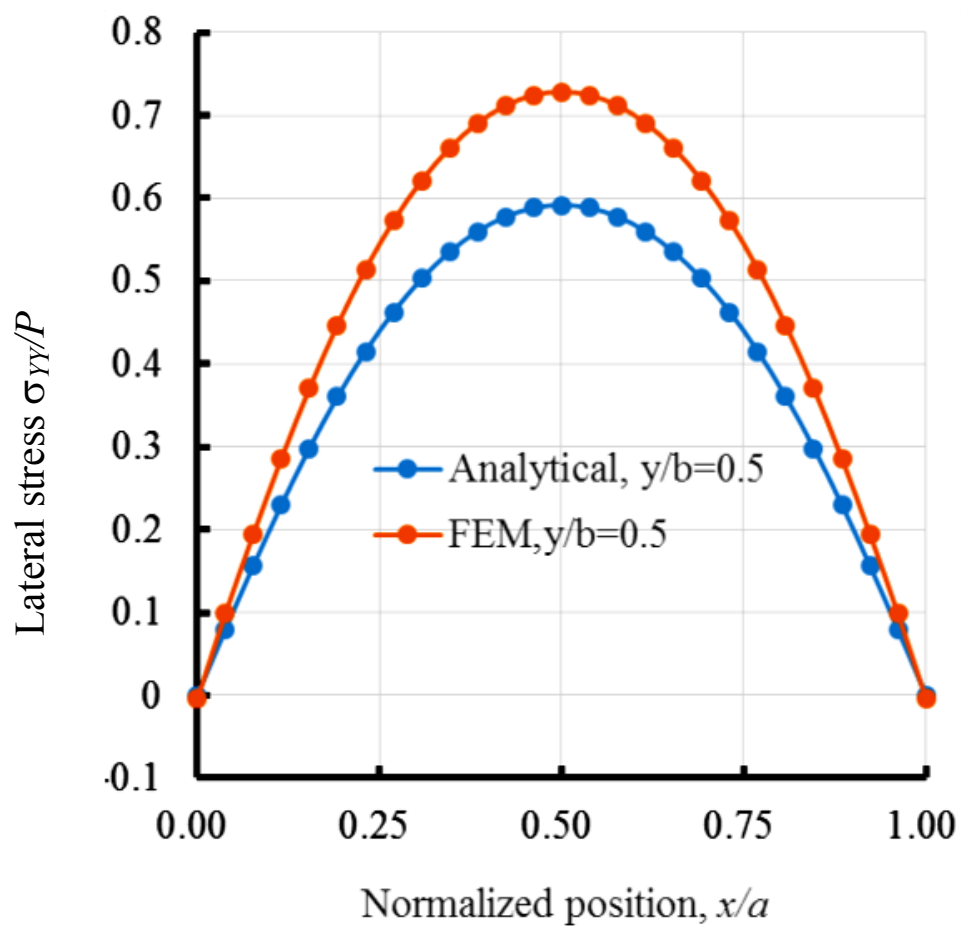

4(b) 


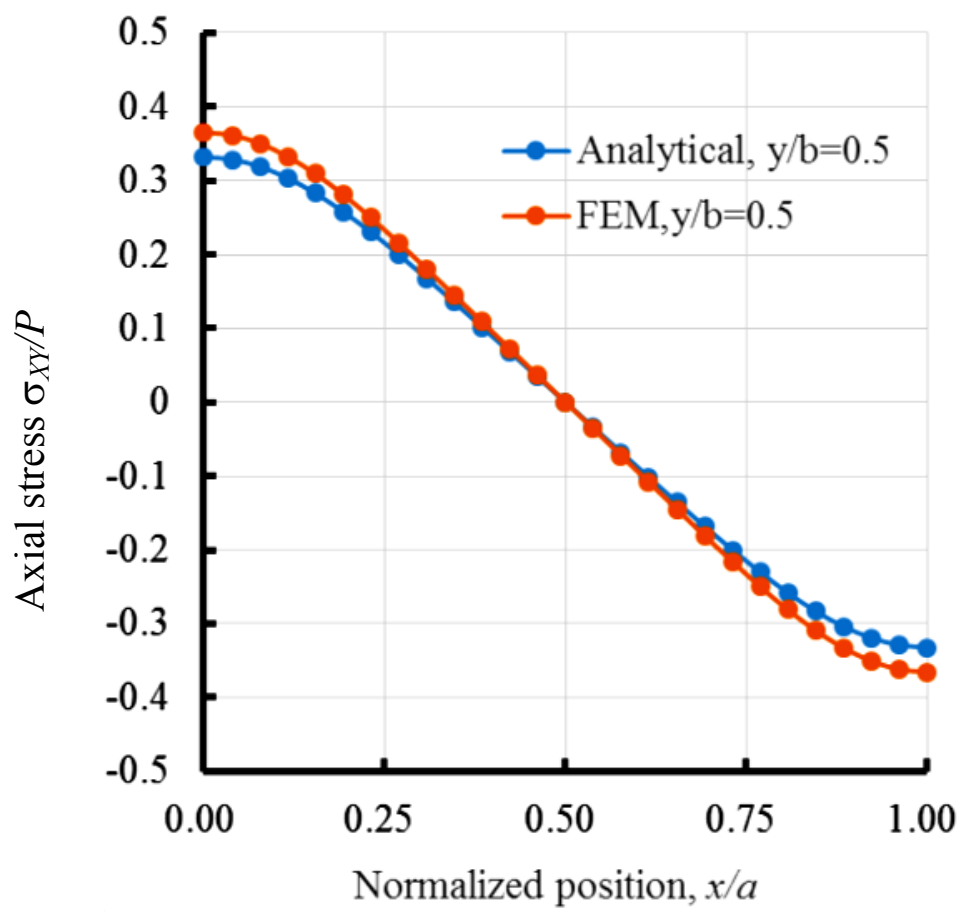

$4(c)$

Fig.4. Comparison of the displacement components: (a) axial stress; (b) lateral stress; (c) shear stress at the section $y / b=0.5$ of the duralumin plate considering plane stress condition shown in Fig. 1 as a function of $x / a$ obtained by the analytical and finite element method.

\section{Conclusions}

The axial stress and lateral displacement components at different sections of the plate for both of the problems for the plane strain condition is higher than that of the plane stress condition. In the case of the plane strain plate, the normal stress along the $\mathrm{z}$ direction for both of the cases is the highest at the loading section and away from the loading edge it gradually decreases. Different stress and displacement components of the problem shown in Fig. 1 for the case of plane stress conditions obtained from the present study and the finite element simulation agree well.

\section{Nomenclature}

$$
\begin{aligned}
\mathrm{E} & - \text { Young's modulus of duralumin } \\
P & - \text { applied normal stress on the boundary of a rectangular plate } \\
u_{x}(x, y) & - \text { lateral displacement component } \\
u_{y}(x, y) & - \text { axial displacement component } \\
v & - \text { Poisson's ratio of a material } \\
\sigma_{x x} & - \text { normal stress component along the } x \text { direction } \\
\sigma_{x y} & - \text { shear stress component along } x y \text { plane } \\
\sigma_{y y} & - \text { normal stress component along the } y \text { direction } \\
\sigma_{z z} & - \text { normal stress component along the } \mathrm{z} \text { direction } \\
\psi & - \text { displacement potential function }
\end{aligned}
$$




\section{References}

[1] Airy G.B. (1863): On the strains in the interior of beams. - Philos. Trans. R. Soc. London, Ser. A 153, pp.49-79.

[2] Love A.E.H. (1892; 1944): A treatise on the mathematical theory of elasticity. - vol.1, Cambridge Univ. Press, Cambridge (1892), Reprinted: Dover, New York (1944)

[3] Filon L.N.G. (1903): On an approximate solution for the bending of a beam of rectangular cross-section under any system of load, with special references to points of concentrated or discontinuous loading. - Philos. Trans. R. Soc. London, Ser. A 201, pp.63-155.

[4] Michell J.H. (1899): On the direct determination of stress in an elastic solid, with application to the theory of plates. - Proc. London Math. Soc., vol.31, pp.100-121.

[5] Michell J.H. (1901): Elementary distributions of plane stress. - Proc. London Math. Soc., vol.32, pp.35-61.

[6] Papkovich P.F. (1937): A derivation the main formulae of the plain problem of the theory of elasticity from the general integral of the Lame equations (in Russian). - Prikl. Mat. Mekh., pp.147-154.

[7] Kolosov G.V. (1935): Application of complex diagrams and theory of functions of complex variable to the theory of elasticity (in Russian). - ONTI, Leningrad-Moscow.

[8] Golovin K.H. (1881): One problem in statics of an elastic body (in Russian). - Izvestiya St. Peterburg Prakt. Tekhnol. Inst., vol.3, pp.373-410.

[9] Muskhelishelishvili N.I. (1953): Some basic problems of the mathematical theory of elasticity. - Noordhoff, Groningen.

[10] Vigak V.M. (1997): Solution of two-dimensional problems of elasticity and thermoelasticity for a rectangular region. - Journal of Mathematical Sciences, vol..86, pp.2537-2542.

[11] Vihak V.M., Yuzvyak M.Yo. (2001): Key continuity equations in stresses for axisymmetric problems of elasticity and thermoelasticity. - Journal of Mathematical Sciences, vol.107, pp.3659-3665.

[12] Vihak V.M. (1995): The solution of the elasticity and thermoelasticity problem in stresses. - Integral Transformations and Their Applications to Boundary Problems, vol.9, pp.34-122.

[13] Vihak V., Tokovyi Y. and Rychahivskyy A. (2002): Exact solution of the plane problem of elasticity in a rectangular region. - Journal of Computational and Applied Mechanics, vol.3, pp.193-206.

[14] Vihak V.M. (1997): Construction of a solution of the plane problem of elasticity and thermoelasticity for orthotropic materials. - Mat. Metody Fiz.-Mekh. Polya, vol.40, pp.24-29.

[15] Parton V.Z. and Perlin P.I. (1981): Methods of Mathematical Theory of Elasticity [in Russian]. - Moscow: Nauka.

[16] Grinchenko V.T. (1978): Equilibrium and Steady Oscillations of Finite Elastic Bodies [in Russian]. - Kiev: Naukova Dumka.

[17] Vihak V.M. (1996): Solution of the two-dimensional problems of elasticity and thermoelasticity for rectangular domains. Mat. Met. Fiz.-Mekh. Polya, vol.39, pp.19-25.

[18] Vihak V.M. and Tokovyi Yu.V. (2002): Investigation of the plane stressed state in a rectangular domain. Materials Science, vol.38, pp.230-237.

[19] Southwell R.V. (1945): On relaxation methods: A mathematics for engineering science, (Bakerian lecture), Proc. R. Soc. London, Ser. A 184, pp.253-288.

[20] Fox L. (1947): Mixed boundary conditions in the relaxational treatment of biharmonical problems (plane strain or stress). - Proc. R. Soc. London, Ser. A 239, pp.419-460.

[21] Meleshko V.V. (2003): Selected topics in the history of the two dimensional biharmonic problem. - Appl. Mech. Rev., vol.56, pp.33-85. 
[22] Odgen R.W. and Isherwood D.A. (1979): Solution of some finite plane strain problems for compressible elastic solids. - J. Mechanics Appl. Math., vol.31, No.2, pp.219-249.

[23] Uddin M.W. (1966): Finite-difference solution of two-dimensional elastic problems with mixed boundary conditions. - M.Sc. Thesis, Carleton University, Canada.

[24] Patnaik S.N. (1986): The variational energy formulation for the integrated force method. - AIAA, J., vol.24, No.1, pp.129-137.

[25] Patnaik S.N., Pai S.S. and Hopkins D.A. (2003): Compatibility condition in theory of solid mechanics (Elasticity, Structures, and Design Optimization). - Appl. Mech. Rev., vol.56, pp.33-85.

[26] Ahmed S.R., Nath S.K.D. and Uddin M.W. (2005): Optimum shapes of tire-treads for avoiding lateral slippage between tires and roads. - International Journal for Numerical Methods in Engineering, vol.64, pp.729-750.

[27] Ahmed S.R. and Nath S.K.D. (2009): A simplified analysis of the tire-tread contact problem using displacement based finite-difference technique. - Computer Modelling in Engineering and Sciences, vol.44, pp.35-63.

[28] Nath S.K.D., Ahmed S.R. and Kim S-G. (2010): On the displacement potential solution of plane problems of structural mechanics with mixed boundary conditions. - Archive of Applied Mechanics, Published online.

[29] Timoshenko S. and Goodier J.N. (1951): Theory of Elasticity. - $2^{\text {nd }}$ ed. New York: McGraw-Hill.

[30] Sadd M.H. (2005): Elasticity: Theory, Applications, Numerics. - USA and UK: Elsevier Academic Press.

Received: September 17, 2016

Revised: March 17, 2017 